\title{
A Comparative Study of Relaying Schemes with Decode and Forward over Nakagami- $m$ Fading Channels
}

\author{
George C. Alexandropoulos, ${ }^{1}$ Agisilaos Papadogiannis, ${ }^{2}$ and Paschalis C. Sofotasios ${ }^{3}$ \\ ${ }^{1}$ Broadband Wireless and Sensor Networks Laboratory, Athens Information Technology, $19.5 \mathrm{~km}$ Markopoulo Avenue, Peania, \\ 19002 Athens, Greece \\ ${ }^{2}$ Department of Signals and Systems, Chalmers University of Technology, 41296 Gothenburg, Sweden \\ ${ }^{3}$ School of Electronic and Electrical Engineering, University of Leeds, Leeds LS2 9JT, UK \\ Correspondence should be addressed to George C. Alexandropoulos, alexandg@ieee.org
}

Received 30 March 2011; Revised 28 June 2011; Accepted 4 August 2011

Academic Editor: W. Hamouda

Copyright () 2011 George C. Alexandropoulos et al. This is an open access article distributed under the Creative Commons Attribution License, which permits unrestricted use, distribution, and reproduction in any medium, provided the original work is properly cited.

\begin{abstract}
Although relaying can be very beneficial for wireless systems, understanding which relaying schemes can achieve specific performance objectives under realistic fading is crucial. In this paper we present a general framework for modeling and evaluating the performance of dual-hop decode-and-forward (DF) relaying schemes over independent and not necessarily identically distributed (INID) Nakagami- $m$ fading channels. We obtain closed-form expressions for the statistics of the instantaneous output signal-to-noise ratio of repetitive transmission with selection diversity. Furthermore, we present a unified statistical overview of other three significant relaying schemes with DF, one based on repetitive transmission with maximal-ratio diversity and the other two based on relay selection (RS). To compare the considered schemes, we present closed-form and analytical expressions for the outage probability and the average symbol error probability under various modulation methods, respectively. Importantly, it is shown that when the channel state information for RS is perfect, RS-based schemes always outperform repetitive ones. Furthermore, when the direct link between the source and the destination nodes is sufficiently strong, relaying may not result in any gains, and it should be switched off.
\end{abstract}

\section{Introduction}

The significance of multiple-input multiple-output (MIMO) techniques for modern wireless systems has been well appreciated. Multiple collocated antennas can improve transmission reliability and the achievable capacity through diversity, spatial multiplexing, and/or interference suppression $[1,2]$. However, the cost of mobile devices is proportional to their number of antennas, and this creates a serious practical limitation for the use of MIMO [3]. Cooperative diversity is a promising new avenue which allows cooperation amongst a number of wireless nodes which effectively profit from MIMO techniques without requiring multiple collocated antennas [4-6].

Dual-hop cooperative diversity entails that the transmission of the source node towards a destination node is assisted by one or more relay nodes which can be seen to form a conceptual MIMO array [4]. Relay nodes can either be fixed, being part of the system infrastructure, or mobile, that is, mobile nodes that relay signals intended for other mobile nodes $[7,8]$. Cooperative diversity can improve the transmission reliability and the achievable capacity, and it can extend coverage. Essentially, it can achieve diversity gains and has the additional advantage over conventional MIMO that the remote cooperating antennas experience very low or inexisting correlation [4, 9-11]. The performance of a system exploiting cooperative diversity depends on the employed relaying protocol and scheme, that is, the way of utilizing relay nodes [4, 9-19]. Consequently, it is crucial to gain insights on which relay scheme is most suitable for achieving a particular objective; some common objectives are the minimization of the outage probability $(\mathrm{OP})$ or the average symbol error probability (ASEP) [20,21].

In the present work, we consider the decode-and-forward (DF) relaying protocol assuming that the message transmitted by the source is decoded and retransmitted to 
the destination by one or more relays in a dual-hop fashion. We take into account four relaying schemes, two based on repetitive transmission and the other two based on relay selection (RS). According to repetitive transmission, all relays that decode the transmitted symbol by the source retransmit it repetitively to the destination node which employs diversity techniques to combine the different received signal copies $[22,23]$. One version of RS entails that, amongst the relays that have decoded the source's symbol, only the node with the strongest relay-to-destination channel is selected to retransmit it to the destination $[9,24]$. Another version of RS utilizes the best relay only when it achieves greater capacity than the direct source-to-destination transmission [10,21]. In the literature the performance of such schemes has been analyzed over independent and identically distributed (IID) Rayleigh fading channels $[9,22,24]$. Recently, the Nakagami$m$ fading model has received a lot of attention as it can describe more accurately the fading process and helps in understanding the gains of cooperative diversity [20, 21, 25-31]. In [26], closed-form expressions for the OP of repetitive transmission with maximal-ratio diversity (MRD) have been derived under independent and not necessarily identically distributed (INID) Nakagami- $m$ fading, whereas for the same fading conditions the OP and ASEP of the aforementioned RS-based schemes have been analyzed in [21]. However, there has not been a complete study that addresses the question of which relaying scheme is preferable and under which channel conditions.

In this paper, we present a general analytical framework for modeling and evaluating performance of relaying schemes with DF under INID Nakagami- $m$ fading channels. More specifically, we obtain closed-form expressions for the OP and ASEP of repetitive transmission when selection diversity (SD) is employed at the destination node. Further to this, we present a comparative study in terms of OP and ASEP performance of RS-based and repetitive transmission schemes. We conclude that the RS-based transmission always performs better in terms of OP and ASEP than repetitive transmission when channel state information (CSI) for RS is perfect. In addition, when the direct source-to-destination link is sufficiently strong, relaying should be disabled when the objective is the minimization of OP. Although RS requires only two time slots for transmission (the repetitive scheme needs as many time slots as the number of decoding relays), its performance heavily relies on the quality of CSI for RS.

The remainder of this paper is structured as follows. Section 2 outlines the system and channel models. Section 3 presents closed-form expressions for the statistics of the instantaneous output SNR of the considered DF relaying schemes over INID Nakagami- $m$ fading channels. In Section 4 closed-form and analytical expressions are derived for the OP and ASEP performance, respectively, of all relaying schemes. Section 5 contains numerical results and relevant discussion, whereas Section 6 concludes the paper.

Notations. Throughout this paper, $|\mathbb{A}|$ represents the cardinality of the set $\mathbb{A}$, and $\mathbb{E}\langle\cdot\rangle$ denotes the expectation operator. $\operatorname{Pr}\{\cdot\}$ denotes probability, $\mathbb{L}^{-1}\{\cdot ; \cdot\}$ denotes the inverse
Laplace transform, and $X \sim \mathcal{C} \mathcal{N}\left(\mu, \sigma^{2}\right)$ represents a random variable (RV) following the complex normal distribution with mean $\mu$ and variance $\sigma^{2} . \Gamma(\cdot)$ is the Gamma function [32, equation (8.310/1)], and $\boldsymbol{\Gamma}(\cdot, \cdot)$ is the lower incomplete Gamma function [32, equation $(8.350 / 1)]$. Moreover, $\delta(\cdot)$ is the Dirac function, $u(\cdot)$ is the unit step function, and $\delta(\cdot, \cdot)$ is the Kronecker Delta function.

\section{System and Channel Model}

We consider a dual-hop cooperative wireless system, as illustrated in Figure 1, consisting of $L+2$ wireless nodes: one source node $S$, a set $\mathbb{A}$ of $L$ relay nodes, each denoted by $R_{k}, k=1,2, \ldots, L$, and one destination node $D$. All $R_{k}$ 's are assumed to operate in half-duplex mode; that is, they cannot transmit and receive simultaneously, and node $D$ is assumed to possess perfect $S \rightarrow D$ and $R_{k} \rightarrow D$, for all $k$, CSI. We consider orthogonal DF (ODF) relaying [4] entailing that each $R_{k}$ that successfully decodes $S$ 's signal retransmits it to $D$; during each $R_{k}$ 's transmission to $D$, node $S$ remains silent. Repetitive transmission [4] requires $L+1$ time slots to forward $S$ 's signal to $D$ in a predetermined order, whereas only two time slots are needed for RS-based transmission [9]. In both transmission strategies during the first time slot, $S$ broadcasts its signal to all $R_{k}$ 's and also to $D$. Considering quasi-static fading channels, the received signal at $R_{k}$ 's and $D$ in the first time slot can be mathematically expressed as

$$
\begin{aligned}
& y_{D}^{(1)}=h_{S D} s+n_{S D}, \\
& y_{R_{k}}=h_{S R_{k}} s+n_{S R_{k}},
\end{aligned}
$$

where $h_{S D}$ and $h_{S R_{k}}$ denote the $S \rightarrow D$ and $S \rightarrow R_{k}$ complexvalued channel coefficients, respectively, and $s$ denotes the transmitted complex message symbol with average symbol energy $E_{s}$. Moreover, the notations $n_{S D}$ and $n_{S R_{k}}$ in (1) represent the additive white Gaussian noise (AWGN) at $D$ and $R_{k}$, respectively, with $n_{S D}, n_{S R_{k}} \sim \mathcal{C} \mathcal{N}\left(0, N_{0}\right)$. For both $n_{S D}$ and $n_{S R_{k}}$, it is assumed that they are statistically independent of $s$.

Let us assume that a set $\mathbb{B} \subseteq \mathbb{A}$ contains the relay nodes that have successfully decoded $S$ 's signal during the first time slot of transmission. Repetitive transmission requires that $L$ more time slots are used for $R_{k}$ 's belonging to $\mathbb{B}$ to forward $s$ to $D$; each $R_{k}$ retransmits $s$ during the $k$ th time slot (Note that the assignment of each time slot to $R_{k}$ 's is performed in a predetermined order [4]. Thus, due to different $R_{k} \rightarrow D$ for all $k$ channel conditions and ODF relaying, there might be some unused time slots.). Hence, for quasi-static fading, the received signal at $D$ at the $k$ th time slot can be expressed as

$$
y_{D}^{(k)}=h_{R_{k} D} s+n_{R_{k} D}
$$

with $h_{R_{k} D}$ representing the $R_{k} \rightarrow D$ complex-valued channel coefficient and $n_{R_{k} D} \sim \mathcal{C} \mathcal{N}\left(0, N_{0}\right)$ the AWGN that is again assumed statistically independent of $s$.

When RS-based transmission is used, one time slot is needed for the relay node $R_{\text {best }}$ with the most favourable $R_{\text {best }} \rightarrow D$ channel conditions to forward $S$ 's signal to $D$. 


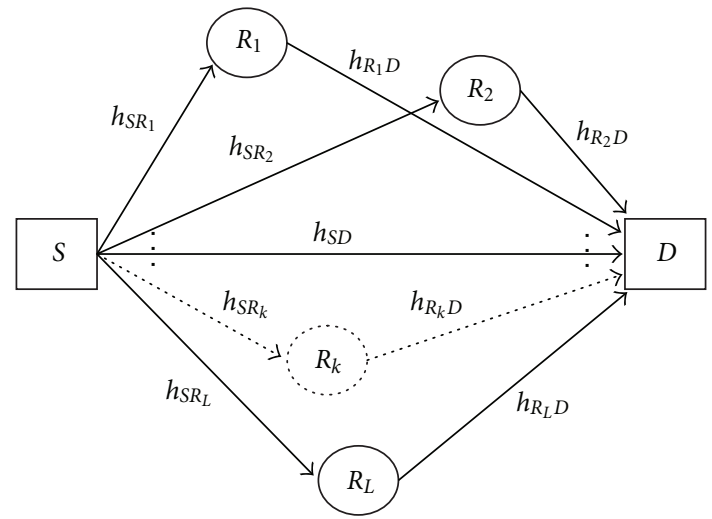

Figure 1: An illustration of a dual-hop cooperative wireless system with $L+2$ wireless nodes: a source node $S, L$ relay nodes $R_{k}, k=$ $1,2, \ldots, L$, and a destination node $D$.

Thus, for this transmission strategy and during the second time slot, the received signal at $D$ for quasi-static fading can be expressed as

$$
y_{D}^{(2)}=h_{R_{\text {best }} D} s+n_{R_{\text {best }} D},
$$

where $h_{R_{\text {best }} D}$ and $n_{R_{\text {best }} D} \sim \mathcal{C} \mathcal{N}\left(0, N_{0}\right)$ denote the $R_{k} \rightarrow D$ complex-valued channel coefficient and the AWGN, respectively. As in (1) and (2), it is assumed that $n_{R_{\text {best }} D}$ is statistically independent of $s$.

The quasi-static fading channels $h_{S D}, h_{S R_{k}}$, and $h_{R_{k} D}$, for all $k$, and $h_{R_{\text {best }} D}$ are modeled as INID Nakagami- $m$ RVs [33]. Let $\gamma_{0}=\left|h_{S D}\right|^{2} E_{s} / N_{0}$ and $\gamma_{k}=\left|h_{R_{k} D}\right|^{2} E_{s} / N_{0}, k=$ $1,2, \ldots, L$, denote the instantaneous received SNRs of the $S \rightarrow D$ and $R_{k} \rightarrow D$ link, respectively, with corresponding average values represented by $\bar{\gamma}_{0}=\mathbb{E}\left\langle\left|h_{S D}\right|^{2}\right\rangle E_{s} / N_{0}$ and $\bar{\gamma}_{k}=\mathbb{E}\left\langle\left|h_{R_{k} D}\right|^{2}\right\rangle E_{s} / N_{0}$, respectively. Clearly, each $\gamma_{\ell}, \ell=$ $0,1, \ldots, L$, is gamma distributed with probability density function (PDF) given by [34, Table 2.2]

$$
f_{\gamma_{\ell}}(x)=\frac{C_{\ell}^{m_{\ell}}}{\Gamma\left(m_{\ell}\right)} x^{m_{\ell}-1} \exp \left(-C_{\ell} x\right),
$$

where $m_{\ell} \geq 1 / 2$ denotes the Nakagami- $m$ fading parameter and $C_{\ell}=m_{\ell} / \bar{\gamma}_{\ell}$. Integrating (4), the cumulative distribution function (CDF) of each $\gamma_{e}$ is easily obtained as

$$
F_{\gamma_{\ell}}(x)=\frac{\Gamma\left(m_{\ell}, C_{\ell} x\right)}{\Gamma\left(m_{\ell}\right)} .
$$

The PDFs and CDFs of the instantaneous received SNRs of the first hop, $\gamma_{L+k}=\left|h_{S R_{k}}\right|^{2} E_{\mathrm{S}} / N_{0}$ for all $k$, are given using (4) and (5) by $f_{\gamma_{L+k}}(x)$ and $F_{\gamma_{L+k}}(x)$, respectively. The fading parameters and average SNRs are denoted by $m_{L+k}$ and $\bar{\gamma}_{L+k}=\mathbb{E}\left\langle\left|h_{S R_{k}}\right|^{2}\right\rangle E_{s} / N_{0}$, respectively.

\section{Statistics of ODF Relaying Schemes}

Relay nodes that decode the transmitted signal from $S$ constitute the decoding set $\mathbb{B}$. Based on $[4,24]$, for both transmission strategies the elements of $\mathbb{B}$ are obtained as

$$
\mathbb{B}=\left\{R_{k} \in \mathbb{A}: \log _{2}\left(1+\gamma_{L+k}\right) \geq \alpha \mathcal{R}\right\},
$$

where $\mathcal{R}$ is $S$ 's transmit rate and $\alpha=L+1$ for repetitive transmission, whereas $\alpha=2$ for RS-based transmission. Hence, the probability that $R_{k}$ does not belong to $\mathbb{B}$ is easily obtained as

$$
\mathcal{P}_{k}=\operatorname{Pr}\left[\gamma_{L+k}<2^{\alpha \mathcal{R}}-1\right]=F_{\gamma_{L+k}}\left(2^{\alpha \mathcal{R}}-1\right) .
$$

By plugging (5) into (7), we obtain the following closed-form expression for $\mathcal{P}_{k}$ in INID Nakagami- $m$ fading:

$$
\mathcal{P}_{k}=\frac{\Gamma\left[m_{L+k}, C_{L+k}\left(2^{\alpha \mathcal{R}}-1\right)\right]}{\Gamma\left(m_{L+k}\right)} .
$$

To analyze the performance of ODF relaying schemes, the $S \rightarrow D$ direct channel and the $S \rightarrow R_{k} \rightarrow D$, for all $k$, relay-assisted channels are effectively considered as $L+1$ paths between $S$ and $D[22,26]$. Let the zeroth path represent the $S \rightarrow D$ direct link and the $k$ th path the $S \rightarrow R_{k} \rightarrow D$ cascaded link. We define the instantaneous received SNR at $D$ related to these paths as $g_{0}$ and $g_{k}$, respectively. By plugging (4) into [22, equation (4)], the PDFs of $g_{\ell}$ 's, $\ell=0,1, \ldots, L$, are given by

$$
f_{g \ell}(x)=\mathcal{P}_{\ell} \delta(x)+\frac{\left(1-\mathcal{P}_{\ell}\right) C_{\ell}^{m_{\ell}}}{\Gamma\left(m_{\ell}\right)} x^{m_{\ell}-1} \exp \left(-C_{\ell} x\right),
$$

where $\mathcal{P}_{0}$ is the probability that $D$ belongs to $\mathbb{B}$. Clearly, the direct $S \rightarrow D$ path is not linked via a relay; that is, $\mathcal{P}_{0}=0$, yielding $f_{g_{0}}(x)=f_{y_{0}}(x)$. Integrating (9) and using (5) yields the following expression for the CDF of the $\ell$ th cascaded path:

$$
F_{g_{\ell}}(x)=\mathcal{P}_{\ell} u(x)+\left(1-\mathcal{P}_{\ell}\right) \frac{\Gamma\left(m_{\ell}, C_{\ell} x\right)}{\Gamma\left(m_{\ell}\right)} .
$$

Note again that for the direct $S \rightarrow D$ path $F_{g_{0}}(x)=F_{\gamma_{0}}(x)$.

3.1. Repetitive Transmission. The incoming signals at $D$ sent by $S$ and $R_{k} \in \mathbb{B}$, for all $k$, may be combined using a timediversity version of MRD [4] and SD [23]. In particular, $D$ combines $S$ 's signal received in the first time slot with the signals received from $R_{k} \in \mathbb{B}$, for all $k$, in the $L$ subsequent time slots using either MRD or SD.

3.1.1. Repetitive with MRD. With MRD the instantaneous SNR at $D$ 's output is expressed as

$$
g_{\text {end }}=g_{0}+\sum_{i=1}^{|\mathbb{B}|} g_{i}
$$

Since $g_{\ell}$ 's, $\ell=0,1, \ldots, L$, are independent, the momentgenerating function (MGF) of $g_{\text {end }}$ can be easily obtained as the product of the MGFs of $g_{\ell}$ 's. As shown in [26] for INID Nakagami- $m$ fading, using (9) and the definition of the MGF of $g_{k}, k=1,2, \ldots, L$,

$$
\mathcal{M}_{g_{k}}(s)=\int_{0}^{\infty} \exp (-s x) f_{g_{k}}(x) d x,
$$


yields $\mathcal{M}_{g_{k}}(s)=\mathcal{P}_{k}+\left(1-\mathcal{P}_{k}\right) C_{k}^{m_{k}}\left(s+C_{k}\right)^{-m_{k}}$. Similarly, using (4) the MGF of $g_{0}$ is easily obtained as $\mathcal{M}_{g_{0}}(s)=$ $C_{0}^{m_{0}}\left(s+C_{0}\right)^{-m_{0}}$. Hence, the following closed-form expression for the MGF of $g_{\text {end }}$ in INID Nakagami- $m$ is deduced:

$$
\begin{aligned}
& \mathcal{M}_{g_{\text {end }}}(s) \\
& =C_{0}^{m_{0}}\left(s+C_{0}\right)^{-m_{0}} \prod_{k=1}^{L}\left[\mathcal{P}_{k}+\left(1-\mathcal{P}_{k}\right) C_{k}^{m_{k}}\left(s+C_{k}\right)^{-m_{k}}\right] .
\end{aligned}
$$

Using the MGF-based approach [34], the CDF of $g_{\text {end }}$ can be obtained as

$$
F_{g_{\text {end }}}(x)=\mathbb{L}^{-1}\left\{\frac{\mathcal{M}_{g_{\text {end }}}(s)}{s} ; x\right\} .
$$

By plugging (13) into (14) and similarly with [26], we obtain the following closed-form expression for $F_{g_{\text {end }}}(x)$ of repetitive transmission with MRD over INID Nakagami- $m$ fading with integer $m_{\ell}$ 's and distinct $C_{\ell}$ 's:

$$
\begin{aligned}
F_{g_{\text {end }}}(x)= & \left(\prod_{\ell=0}^{L} \mathcal{P}_{\ell}\right) \\
& \times\left\{1+\sum_{\left\{\lambda_{k}\right\}_{k=0}^{L}}\left(\prod_{n=0}^{k} \frac{1-\mathcal{P}_{\lambda_{n}}}{\mathcal{P}_{\lambda_{n}}} C_{\lambda_{n}}^{m_{\lambda_{n}}}\right)\right. \\
& \left.\times \sum_{p=0}^{k} \sum_{q=1}^{m_{\lambda_{p}}} \frac{\psi_{p}}{C_{\lambda_{p}}^{q}}\left[1-\exp \left(-C_{\lambda_{p}} x\right) \sum_{\ell=0}^{q-1} \frac{\left(C_{\lambda_{p}} x\right)^{\ell}}{\ell !}\right]\right\} .
\end{aligned}
$$

The symbol $\sum_{\left\{\alpha_{i}\right\}_{i=k}^{I}}$ is used for short-hand representation of multiple summations $\sum_{i=\kappa}^{I} \sum_{\alpha_{\kappa}=\kappa}^{I-i+\kappa} \sum_{\alpha_{\kappa+1}=\alpha_{\kappa}+1}^{I-i+\kappa+1} \cdots \sum_{\alpha_{i}=\alpha_{i-1}+1}^{I}$ and $\psi_{p}=\left.\Psi_{p}(s)^{\left(m_{\lambda_{p}}-q\right)}\right|_{s=-C_{\lambda_{p}}} /\left(m_{\lambda_{p}}-q\right)$ ! with $\Psi_{p}(s)=(s+$ $\left.C_{\lambda_{p}}\right)^{m_{\lambda_{p}}} \prod_{n=0}^{k}\left(s+C_{\lambda_{n}}\right)^{-m_{\lambda_{n}}}$. For $C_{\ell}=C$, for all $\ell$, with arbitrary values for $m_{\ell}$ 's and following a similar analysis as for the derivation of (15), a closed-form expression for $F_{g_{\text {end }}}(x)$ can be obtained as

$$
\begin{aligned}
& F_{g_{\text {end }}}(x) \\
& =\left(\prod_{\ell=0}^{L} \mathcal{P}_{\ell}\right)\left\{1+\sum_{\left\{\lambda_{k}\right\}}\left(\prod_{k=0}^{k} \frac{1-\mathcal{P}_{\lambda_{n}}}{\mathcal{P}_{\lambda_{n}}}\right) \frac{\boldsymbol{\Gamma}\left(\sum_{n=0}^{k} m_{\lambda_{n}}, C x\right)}{\Gamma\left(\sum_{n=0}^{k} m_{\lambda_{n}}\right)}\right\} .
\end{aligned}
$$

3.1.2. Repetitive with $S D$. An alternative to $M R D$ is a timediversity version of SD allowing $D$ to combine the received signals from $S$ and $R_{k} \in \mathbb{B}$ for all $k$ [23]. With this diversity technique, the instantaneous SNR at D's output is given by

$$
g_{\text {end }}=\max _{R_{k} \in \mathbb{C}} g_{k}
$$

where $\mathbb{C}=\{S\} \cup \mathbb{B}$. The $g_{\ell}$ 's, $\ell=0,1, \ldots, L$, are assumed to be independent; therefore, $F_{g_{\text {end }}}(x)$ of (17) can be easily obtained as the product of the CDFs of $g_{\ell}$ 's. With the use of (5) and (10) for $g_{0}$ and $g_{k}$ for all $k=1,2, \ldots, L$, respectively, a closedform expression for $F_{g_{\text {end }}}(x)$ of repetitive transmission with SD under INID Nakagami- $m$ fading channels can be derived as

$$
F_{g_{\text {end }}}(x)=F_{\gamma_{0}}(x) F_{g_{\text {best }}}(x),
$$

where $F_{g_{\text {best }}}(x)$ is the CDF of the instantaneous SNR of the $R_{\text {best }} \rightarrow D$ channel, that is, of

$$
g_{\text {best }}=\max _{R_{k} \in \mathbb{B}} g_{k},
$$

which is easily obtained using (10) for INID Nakagami- $m$ fading as

$$
F_{g_{\text {best }}}(x)=\prod_{k=1}^{L}\left[\mathcal{P}_{k} u(x)+\left(1-\mathcal{P}_{k}\right) \frac{\Gamma\left(m_{k}, C_{k} x\right)}{\Gamma\left(m_{k}\right)}\right] .
$$

Differentiating (18), the PDF of $g_{\text {end }}$ is given by

$$
f_{g_{\text {end }}}(x)=f_{\gamma_{0}}(x) F_{g_{\text {best }}}(x)+F_{\gamma_{0}}(x) f_{g_{\text {best }}}(x),
$$

where $f_{g_{\text {best }}}(x)$ is the PDF of $g_{\text {best }}$. To obtain an expression for $f_{g_{\text {best }}}(x)[21]$, we first use [32, equation $\left.(8.352 / 1)\right]$ to obtain (20) for integer $m_{\ell}$ 's yielding

$$
\begin{aligned}
& F_{g_{\text {best }}}(x) \\
& =\prod_{k=1}^{L}\left\{\mathcal{P}_{k} u(x)+\left(1-\mathcal{P}_{k}\right)\left[1-\exp \left(-C_{k} x\right) \sum_{i=0}^{m_{k}-1} \frac{\left(C_{k} x\right)^{i}}{i !}\right]\right\} .
\end{aligned}
$$

Then, differentiating (22) and using the formula

$$
\prod_{i=\kappa}^{I}\left(\chi_{i}+\psi_{i}\right)=\prod_{i=\kappa}^{I} \chi_{i}+\sum_{\left\{\alpha_{i}\right\}_{i=\kappa}^{I}} \prod_{s=\kappa}^{i} \psi_{\alpha_{s}} \prod_{\substack{t=\kappa \\ t \neq\left\{\alpha_{u}\right\}_{u=\kappa}^{i}}}^{I} \chi_{t},
$$

a closed-form expression for $f_{g_{\text {best }}}(x)$ over INID Nakagami- $m$ fading channels with integer $m_{\ell}$ 's is obtained as [21, equation (6)]

$$
\begin{aligned}
& f_{g_{\text {best }}}(x) \\
& =\left(\prod_{k=1}^{L} \mathcal{P}_{k}\right)\left\{\delta(x)+\sum_{\left\{\lambda_{k}\right\}_{k=1}^{L}} \sum_{\left\{\mu_{j}\right\}_{j=1}^{k}}(-1)^{j}\left(\prod_{n=1}^{k} \frac{1-\mathcal{P}_{\lambda_{n}}}{\mathcal{P}_{\lambda_{n}}}\right)\right. \\
& \times\left[-\left(\sum_{p=1}^{j} C_{\lambda_{\mu_{p}}}\right) \exp \left(-x \sum_{p=1}^{j} C_{\lambda_{\mu_{p}}}\right)\right. \\
& +\sum_{\left\{v_{i}\right\}_{i=1}^{j}}\left(\sum_{s=1}^{p} i_{v_{s}}\right)\left(\prod_{s=1}^{p} \sum_{i_{v_{s}}=1}^{m_{\lambda \mu_{s}}-1} \frac{C_{\lambda_{\mu_{\nu_{s}}}}^{i_{\nu_{s}}}}{i_{\nu_{s}} !}\right) \\
& \left.\left.\times x^{\sum_{s=1}^{p} i_{v_{s}}-1} \exp \left(-x \xi_{p, j}\right)\left(1-\frac{\xi_{p, j} x}{\sum_{s=1}^{p} i_{v_{s}}}\right)\right]\right\} .
\end{aligned}
$$


In (24), parameters $\xi_{p, j}$ 's, with $p$ and $j$ being positive integers, are given by

$$
\xi_{p, j}=\sum_{s=1}^{p} C_{\lambda_{\mu v_{s}}}+\sum_{\substack{t=1 \\ t \neq\left\{v_{u}\right\}_{u=1}^{p}}}^{j} C_{\lambda_{\mu t}} .
$$

Using (4) and (5) for integer $m_{\ell}$ 's, that is, after using [32, equation $(8.352 / 1)]$ for expressing $\Gamma(\cdot, \cdot)$ 's, as well as $(22)$ and (24) to (21), and after some algebraic manipulations, we obtain the following novel closed-form expression for $f_{g_{\text {end }}}(x)$ :

$$
\begin{aligned}
& f_{g_{\text {end }}}(x) \\
& =\left(\prod_{k=1}^{L} \mathcal{P}_{k}\right)\left\{\frac{C_{0}^{m_{0}}}{\Gamma\left(m_{0}\right)} x^{m_{0}-1} \exp \left(-C_{0} x\right)\right. \\
& \times\left\{u(x)+\sum_{\left\{\lambda_{k}\right\}_{k=1}^{L}} \sum_{\left\{\mu_{j}\right\}_{j=1}^{k}}\left(\prod_{n=1}^{k} \frac{1-\mathcal{P}_{\lambda_{n}}}{\mathcal{P}_{\lambda_{n}}}\right)\right. \\
& \times\left[\exp \left(-x \sum_{p=1}^{j} C_{\lambda_{\mu_{p}}}\right)-1\right. \\
& \left.\left.+\sum_{\left\{v_{i}\right\}_{i=1}^{j}}\left(\prod_{s=1}^{p} \sum_{i_{v_{s}}=1}^{m_{\lambda_{\mu \nu_{s}}}-1} \frac{C_{\lambda_{\mu \nu_{s}}}^{i_{v_{s}}}}{i_{v_{s}} !}\right) x^{\sum_{s=1}^{p} i_{v_{s}}} \exp \left(-x \xi_{p, j}\right)(-1)^{j}\right]\right\} \\
& +\left[1-\exp \left(-C_{0} x\right) \sum_{i=0}^{m_{0}-1} \frac{\left(C_{0} x\right)^{i}}{i !}\right] \\
& \times\left\{\delta(x)+\sum_{\left\{\lambda_{k}\right\}_{k=1}^{L}} \sum_{\left\{\mu_{j}\right\}_{j=1}^{k}}(-1)^{j}\left(\prod_{n=1}^{k} \frac{1-\mathcal{P}_{\lambda_{n}}}{\mathcal{P}_{\lambda_{n}}}\right)\right. \\
& \times\left[-\left(\sum_{p=1}^{j} C_{\lambda_{\mu p}}\right) \exp \left(-x \sum_{p=1}^{j} C_{\lambda_{\mu p}}\right)\right. \\
& +\sum_{\left\{v_{i}\right\}_{i=1}^{j}}\left(\sum_{s=1}^{p} i_{v_{s}}\right)\left(\prod_{s=1}^{p} \sum_{i_{v_{s}}=1}^{m_{\mu \nu_{s}}-1} \frac{C_{\lambda_{\mu \nu_{s}}}^{i_{\nu_{s}}}}{i_{\nu_{s}} !}\right) x^{\sum_{s=1}^{p} i_{v_{s}}-1} \\
& \left.\left.\times \exp \left(-x \xi_{p, j}\right)\left(1-\frac{\xi_{p, j} x}{\sum_{s=1}^{p} i_{v_{s}}}\right)\right]\right\},
\end{aligned}
$$

which is valid for INID Nakagami- $m$ fading with integer values of $m_{\ell}$ 's and distinct $C_{\ell}$ 's.

To derive the MGF of $g_{\text {end }}$, we plug the $f_{g_{\text {end }}}(x)$ expression of (26) to the definition of the MGF given by (12), that is, after replacing $g_{k}$ with $g_{\text {end }}$ in (12), and use [32, equation $(3.381 / 4)]$ to solve the resulting integrals. In particular, by first deriving using (24) the following closed-form expression for the MGF of $g_{\text {best }}$ in INID Nakagami- $m$ fading with integer values of $m_{\ell}$ 's and distinct $C_{\ell}$ 's:

$$
\begin{aligned}
& \mathcal{M}_{g_{\text {best }}}(s) \\
& =\left(\prod_{k=1}^{L} \mathcal{P}_{k}\right) \\
& \times\left\{1+\sum_{\left\{\lambda_{k}\right\}_{k=1}^{L}} \sum_{\left\{\mu_{j}\right\}_{j=1}^{k}}(-1)^{j}\left(\prod_{n=1}^{k} \frac{1-\mathcal{P}_{\lambda_{n}}}{\mathcal{P}_{\lambda_{n}}}\right)\right. \\
& \times\left\{-\left(\sum_{p=1}^{j} C_{\lambda_{\mu p}}\right)\left(s+\sum_{p=1}^{j} C_{\lambda_{\mu p}}\right)^{-1}\right. \\
& +\sum_{\left\{v_{i}\right\}_{i=1}^{j}}\left(\sum_{s=1}^{p} i_{v_{s}}\right) !\left(\prod_{s=1}^{p} \sum_{i_{v_{s}}=1}^{m_{\mu \nu_{s}}-1} \frac{C_{\lambda_{\mu_{v_{s}}}}^{i_{\nu_{s}}}}{i_{v_{s}} !}\right) \\
& \left.\left.\times\left(s+\xi_{p, j}\right)^{-\sum_{s=1}^{p} i_{v_{s}}}\left[1-\xi_{p, j}\left(s+\xi_{p, j}\right)^{-1}\right]\right\}\right\},
\end{aligned}
$$

a novel closed-form expression for the MGF of $g_{\text {end }}$ of repetitive transmission with SD over INID Nakagami- $m$ fading channels with integer values of $m_{\ell}$ 's and distinct $C_{\ell}$ 's is given by

$$
\begin{aligned}
& \mathcal{M}_{g_{\text {end }}}(s)=\left(\prod_{k=1}^{L} \mathcal{P}_{k}\right) \\
& \times\left\{C _ { 0 } ^ { m _ { 0 } } \left\{\left(s+C_{0}\right)^{-m_{0}}+\sum_{\left\{\lambda_{k}\right\}_{k=1}^{L}} \sum_{\left\{\mu_{j}\right\}_{j=1}^{k}}(-1)^{j}\left(\prod_{n=1}^{k} \frac{1-\mathcal{P}_{\lambda_{n}}}{\mathcal{P}_{\lambda_{n}}}\right)\right.\right. \\
& \times\left[\left(s+C_{0}+\sum_{p=1}^{j} C_{\lambda_{\mu p}}\right)^{-m_{0}}-\left(s+C_{0}\right)^{-m_{0}}\right. \\
& +\sum_{\left\{v_{i}\right\}_{i=1}^{j}}\left(\prod_{s=1}^{p} \sum_{i_{v_{s}}=1}^{m_{\lambda \mu_{\nu_{s}}}-1} \frac{C_{\lambda_{\mu_{\nu_{s}}}}^{i_{v_{s}}}}{i_{v_{s}} !}\right) \frac{\left(m_{0}+\sum_{s=1}^{p} i_{v_{s}}-1\right) !}{\left(m_{0}-1\right) !} \\
& \left.\left.\times\left(s+C_{0}+\xi_{p, j}\right)^{-\left(m_{0}+\sum_{s=1}^{p} i_{v_{s}}\right)}\right]\right\} \\
& +\left\{\sum_{\left\{\lambda_{k}\right\}_{k=1}^{L}} \sum_{\left\{\mu_{j}\right\}_{j=1}^{k}}(-1)^{j}\left(\prod_{n=1}^{k} \frac{1-\mathcal{P}_{\lambda_{n}}}{\mathcal{P}_{\lambda_{n}}}\right)\right. \\
& \times\left\{\left(\sum_{p=1}^{j} C_{\lambda_{\mu p}}\right) \sum_{q=0}^{m_{0}-1} C_{0}^{q}\left(s+C_{0}+\sum_{p=1}^{j} C_{\lambda_{\mu p}}\right)^{-(q+1)}\right.
\end{aligned}
$$




$$
\begin{aligned}
& +\sum_{\left\{v_{i}\right\}_{i=1}^{j}}\left(\sum_{s=1}^{p} i_{v_{s}}\right)\left(\prod_{s=1}^{p} \sum_{i_{v_{s}}=1}^{m_{\lambda_{\nu_{s}}}-1} \frac{C_{\lambda_{\mu \nu_{s}}}^{i_{v_{s}}}}{i_{v_{s}} !}\right) \\
& \times \sum_{q=0}^{m_{0}-1} \frac{C_{0}^{q}}{q !}\left(s+C_{0}+\xi_{p, j}\right)^{-\left(q+\sum_{s=1}^{p} i_{v_{s}}+1\right)} \\
& \left.\left.\times\left(q+\sum_{s=1}^{p} i_{v_{s}}-1\right) !\left[q \xi_{p, j}-\left(s+C_{0}\right) \sum_{s=1}^{p} i_{v_{s}}\right]\right\}\right\}
\end{aligned}
$$$$
+\mathcal{M}_{g_{\text {best }}}(s) \text {. }
$$

It is noted that (28) corrects a typo that appears in [28, equation (7)]. For equal $C_{\ell}$ 's, that is, IID Nakagami- $m$ fading with $m_{\ell}=m$ and $\bar{\gamma}_{\ell}=\bar{\gamma}$ for all $\ell$, following a similar procedure as for the derivation of (28) and using the binomial and multinomial theorems [32, equation (1.111)], we first obtain the following closed-form expression for $\mathcal{M}_{g_{\text {best }}}(s)$ for integer $m$ :

$$
\begin{gathered}
\mathcal{M}_{g_{\text {best }}}(s) \\
=L \mathcal{P}^{L}\left\{\frac{1}{L}+(1-\mathcal{P}) C^{m}(s+C)^{-m}+\sum_{k=1}^{L-1}\left(\begin{array}{c}
L-1 \\
k
\end{array}\right) \frac{(1-\mathcal{P})^{k}}{\mathcal{P}^{k+1}}\right. \\
\times\left\{\mathcal{P}+(1-\mathcal{P}) C^{m}(s+C)^{-m}+\sum_{j=1}^{k}\left(\begin{array}{c}
k \\
j
\end{array}\right)(-1)^{j}\right. \\
\times\left[\mathcal{P}+\sum_{n_{1}+n_{2}+\cdots+n_{m}=j}^{j} \frac{(1-\mathcal{P}) C^{m+\sigma}}{\prod_{j=2}^{m-1}(j !)^{n_{j+1}}} \frac{\prod_{i=1}^{m} n_{i}^{-1}}{(m-1) !}\right. \\
\left.\left.\left.\times[s+(j+1) C]^{-(m+\sigma)}\right]\right\}\right\}
\end{gathered}
$$

where $C=m / \bar{\gamma}, \mathcal{P}=\mathcal{P}_{k}$ for all $k, \sigma=\sum_{i=2}^{m}(i-1) n_{i}$, and symbol $\sum_{n_{1}+n_{2}+\cdots+n_{m}=j}^{j}$ is used for short-hand representation of multiple summations $\sum_{n_{1}=0}^{j} \sum_{n_{2}=0}^{j} \cdots \sum_{n_{m}=0}^{j} \delta\left(\sum_{i=1}^{m} n_{i}, j\right)$. Using (21) for IID Nakagami- $m$ fading, (29) and after some algebraic manipulations, a novel closed-form expression for $\mathcal{M}_{g_{\text {end }}}(s)$ for repetitive transmission with SD in IID Nakagami- $m$ fading channels with integer $m$ is obtained as

$$
\begin{aligned}
\mathcal{M}_{g_{\text {end }}}(s) & L^{L}\left\{C ^ { m } \left\{\left[\frac{1}{L}+(1-\mathcal{P})\right](s+C)^{-m}\right.\right. \\
& -(1-\mathcal{P}) B_{0,1}(s)+\sum_{k=1}^{L-1}\left(\begin{array}{c}
L-1 \\
k
\end{array}\right) \frac{(1-\mathcal{P})^{k}}{\mathcal{P}^{k+1}}
\end{aligned}
$$

$$
\begin{aligned}
& \times\left\{[\mathcal{P}+(1-\mathcal{P})](s+C)^{-m}\right. \\
& -(1-\mathcal{P}) B_{0,1}(s)+\sum_{j=1}^{k}\left(\begin{array}{l}
k \\
j
\end{array}\right)(-1)^{j} \\
& \times\left[\mathcal{P}(s+C)^{-m}+\sum_{n_{1}+n_{2}+\cdots+n_{m}=j}^{j} \frac{(1-\mathcal{P})}{\prod_{j=2}^{m-1}(j !)^{n_{j+1}}}\right. \\
& \left.\left.\left.\times \frac{\prod_{i=1}^{m} n_{i}^{-1}}{(m-1) !}\left\{(s+C)^{-m}-B_{\sigma, j+1}(s)\right\}\right]\right\}\right\} \\
& -C^{m}\left\{(1-\mathcal{P}) B_{0,1}(s)+\sum_{k=1}^{L-1}\left(\begin{array}{c}
L-1 \\
k
\end{array}\right) \frac{(1-\mathcal{P})^{k}}{\mathcal{P}^{k+1}}\right. \\
& \times\left\{(1-\mathcal{P}) B_{0,1}(s)+\sum_{j=1}^{k}\left(\begin{array}{l}
k \\
j
\end{array}\right)(-1)^{j}\right. \\
& \times \sum_{n_{1}+n_{2}+\cdots+n_{m}=j}^{j} \frac{(1-\mathcal{P}) C^{\sigma}}{\prod_{j=2}^{m-1}(j !)^{n_{j+1}}} \frac{\prod_{i=1}^{m} n_{i}^{-1}}{(m-1) !} \\
& \times \sum_{q=0}^{m-1} \frac{C^{q}}{q !(m+\sigma-1) !} \\
& \left.\left.\left.\times(m+q+\sigma-1) ![s+(\lambda+1) C]^{-(m+q)}\right\}\right\}\right\}
\end{aligned}
$$$$
+\mathcal{M}_{g_{\text {best }}}(s)
$$

where function $B_{\kappa, \lambda}(s)$, with $\kappa$ and $\lambda$ being positive integers, is given by

$$
B_{\kappa, \lambda}(s)=\sum_{q=0}^{m+\kappa-1} \frac{(\lambda C)^{q}(m+q-1) !}{q !(m-1) ![s+(\lambda+1) C]^{m+q}} .
$$

3.2. RS-Based Transmission. When RS-based transmission is utilized, RS is first performed to obtain $R_{\text {best }}[11,21,28]$. Relay node $R_{\text {best }}$ is the one experiencing the most favorable $R_{k} \rightarrow D$, for all $k$, channel conditions; that is, its instantaneous SNR is given by (19). Using expressions derived in Section 3.1, we present closed-form expressions for the statistics of a pure RS scheme $[21,24]$ that combines at $D$ the received signals from $S$ and $R_{\text {best }}$ using a time-diversity version of MRD as well as of a rate-selective one $[11,21]$ that utilizes pure RS only if it is beneficial in terms of achievable rate over the direct transmission.

3.2.1. Pure RS. With pure RS, $D$ utilizes MRD to combine the signals from $S$ and $R_{\text {best }}$ [24]; therefore, the instantaneous SNR at D's output is given, using (19), by

$$
g_{\text {end }}=g_{0}+g_{\text {best }}
$$


Similar to the derivation of (13), the MGF of $g_{\text {end }}$ for pure RS can be obtained as the following product:

$$
\mathcal{M}_{g_{\text {end }}}(s)=C_{0}^{m_{0}}\left(s+C_{0}\right)^{-m_{0}} \mathcal{M}_{g_{\text {best }}}(s),
$$

where $\mathcal{M}_{g_{\text {best }}}(s)$ is given by (27) for INID Nakagami- $m$ with integer $m_{\ell}$ 's and distinct $C_{\ell}$ 's, whereas, by (29) for IID Nakagami- $m$ with integer $m_{\ell}=m$ for all $\ell$ and $C_{\ell}=C$. Therefore, by plugging (27) into (33), a closed-form expression for $\mathcal{M}_{\text {gend }}(s)$ of pure RS under INID Nakagami- $m$ fading with integer $m_{\ell}$ 's and distinct $C_{\ell}$ 's is given by [21, equation (7)]

$$
\begin{aligned}
& \mathcal{M}_{g_{\text {end }}}(s) \\
& =C_{0}^{m_{0}}\left(s+C_{0}\right)^{-m_{0}}\left(\prod_{k=1}^{L} \mathcal{P}_{k}\right) \\
& \times\left\{1+\sum_{\left\{\lambda_{k}\right\}_{k=1}^{L}\left\{\sum_{j j}\right\}_{j=1}^{k}}(-1)^{j}\left(\prod_{n=1}^{k} \frac{1-\mathcal{P}_{\lambda_{n}}}{\mathcal{P}_{\lambda_{n}}}\right)\right. \\
& \times\left\{-\left(\sum_{p=1}^{j} C_{\lambda_{\mu p}}\right)\left(s+\sum_{p=1}^{j} C_{\lambda_{\mu p}}\right)^{-1}\right. \\
& +\sum_{\left\{v_{i}\right\}_{i=1}^{j}}\left(\sum_{s=1}^{p} i_{v_{s}}\right) !\left(\prod_{s=1}^{p} \sum_{i_{v_{s}}=1}^{m_{\lambda_{\mu v_{s}}}-1} \frac{C_{\lambda_{\mu_{v_{s}}}}^{i_{v_{s}}}}{i_{v_{s}} !}\right) \\
& \left.\left.\times\left(s+\xi_{p, j}\right)^{-\sum_{s=1}^{p} i_{v_{s}}}\left[1-\xi_{p, j}\left(s+\xi_{p, j}\right)^{-1}\right]\right\}\right\} .
\end{aligned}
$$

For IID Nakagami- $m$ fading channels with integer $m$, by plugging (29) into (33) the following closed-form expression for $\mathcal{M}_{g_{\text {end }}}(s)$ of pure RS is obtained [21, equation (10)]:

$$
\begin{aligned}
& \mathcal{M}_{\text {gend }}(s) \\
& =\frac{L \mathcal{P}^{L} C^{m}}{(s+C)^{m}} \\
& \times\left\{\frac{1}{L}+\frac{(1-\mathcal{P}) C^{m}}{(s+C)^{m}}+\sum_{k=1}^{L-1}\left(\begin{array}{c}
L-1 \\
k
\end{array}\right) \frac{(1-\mathcal{P})^{k}}{\mathcal{P} k+1}\right. \\
& \quad \times\left\{\mathcal{P}+\frac{(1-\mathcal{P}) C^{m}}{(s+C)^{m}}+\sum_{j=1}^{k}\left(\begin{array}{c}
k \\
j
\end{array}\right)(-1)^{j}\right. \\
& \quad \times\left[\mathcal{P}+\sum_{n_{1}+n_{2}+\cdots+n_{m}=j}^{j} \frac{(1-\mathcal{P}) C^{m+\sigma}}{\prod_{j=2}^{m-1}(j !)^{n_{j+1}}} \frac{\prod_{i=1}^{m} n_{i}^{-1}}{(m-1) !}\right. \\
& \left.\left.\left.\quad \times[s+(j+1) C]^{-(m+\sigma)}\right]\right\}\right\} .
\end{aligned}
$$

To obtain $F_{\text {gend }}(x)$ for pure RS under INID Nakagami- $m$ fading with integer $m_{\ell}$ 's and distinct $C_{\ell}$ 's, we plug (34) into (14), and, after some algebraic manipulations, the following closed-form expression is derived [21, equation (8)]:

$$
\begin{aligned}
& F_{g_{\text {end }}}(x) \\
& =\left(\prod_{k=1}^{L} \mathcal{P}_{k}\right) \\
& \times\left\{\mathcal{Z}_{m_{0}}\left(C_{0}, x\right)+\sum_{\left\{\lambda_{k}\right\}_{k=1}^{L}} \sum_{\left\{\mu_{j}\right\}_{j=1}^{k}}(-1)^{j}\left(\prod_{n=1}^{k} \frac{1-\mathcal{P}_{\lambda_{n}}}{\mathcal{P}_{\lambda_{n}}}\right)\right. \\
& \times\left\{-C_{0}^{m_{0}}\left\{x_{2,1}^{(1)} z_{0}\left[\chi_{2}^{(1)}, x\right]+\chi_{2}^{(1)} y_{1}^{(1)}(x)\right\}\right. \\
& +\sum_{\left\{v_{i}\right\}_{i=1}^{j}}\left(\prod_{s=1}^{p} \sum_{i_{v_{s}}=1}^{m_{\lambda_{\nu_{s}}}-1} \frac{C_{\lambda_{\mu \nu_{s}}}^{i_{v_{s}}}}{i_{v_{s}} !}\right) \\
& \left.\left.\times C_{0}^{m_{0}}\left(\sum_{s=1}^{p} i_{v_{s}}\right) !\left[y_{2}^{(2)}(x)-\xi_{p, j} y_{2}^{(3)}(x)\right]\right\}\right\},
\end{aligned}
$$

where $\mathcal{Z}_{t}(c, x)=1-\exp (-c x) \sum_{i=1}^{t-1}(c x)^{i} / i$ !, with $c$ being positive real, and

$$
y_{\kappa}^{(u)}(x)=\sum_{r=1}^{\kappa} \sum_{t=1}^{b_{r}^{(u)}} \mathcal{X}_{r, t}^{(u)}\left[\chi_{r}^{(u)}\right]^{-t} \mathcal{Z}_{t}\left[\chi_{r}^{(u)}, x\right]
$$

for $u=1,2$, and 3 . In the above two equations for all $u$, $\chi_{r, t}^{(u)}=\left.X_{r}^{(u)}(s)^{\left(b_{r}^{(u)}-t\right)}\right|_{s=-\chi_{r}^{(u)}} /\left(b_{r}^{(u)}-t\right)$ ! and $X_{r}^{(u)}(s)=(s+$ $\left.\chi_{r}^{(u)}\right)^{b_{r}^{(u)}} \prod_{j=1}^{2}\left(s+\chi_{j}^{(u)}\right)^{b_{j}^{(u)}}$. Moreover, $\chi_{1}^{(u)}=C_{0}$ for all $u, \chi_{2}^{(1)}=$ $\sum_{p=1}^{j} C_{\lambda_{\mu p}}$, and $\chi_{2}^{(2)}=\chi_{2}^{(3)}=\xi_{p, j}$ as well as $b_{1}^{(u)}=m_{0}$, for all $u, b_{2}^{(1)}=1, b_{2}^{(2)}=\sum_{s=1}^{p} i_{v_{s}}$, and $b_{2}^{(3)}=b_{2}^{(2)}+1$. For $C_{\ell}=C$, for all $\ell$, and integer $m$, and similar to the derivation of (36), by plugging (35) into (14), a closed-form expression for $F_{\text {gend }}(x)$ of pure RS is obtained which is given by [21, equation (11)]

$$
\begin{gathered}
F_{g_{\text {end }}}(x) \\
=L \mathcal{P}^{L}\left\{\frac{\mathcal{Z}_{m}(C, x)}{L}+(1-\mathcal{P}) \mathbb{Z}_{2 m}(C, x)\right. \\
+\sum_{k=1}^{L-1}\left(\begin{array}{c}
L-1 \\
k
\end{array}\right) \frac{(1-\mathcal{P})^{k}}{\mathcal{P}^{k+1}}
\end{gathered}
$$




$$
\begin{aligned}
& \times\left\{\mathcal{P Z}_{m}(C, x)+(1-\mathcal{P}) \mathcal{Z}_{2 m}(C, x)\right. \\
& +\sum_{j=1}^{k}\left(\begin{array}{l}
k \\
j
\end{array}\right)(-1)^{j} \\
& \times\left[\mathcal{P} Z_{m}(C, x)\right. \\
& +\sum_{n_{1}+n_{2}+\cdots+n_{m}=j}^{j} \frac{(1-\mathcal{P}) \prod_{i=1}^{m} n_{i}^{-1} C^{2 m+\sigma}}{(m-1) ! \prod_{j=2}^{m-1}(j !)^{n_{j+1}}} \\
& \left.\left.\left.\times y_{2}^{(4)}(x)\right]\right\}\right\}
\end{aligned}
$$

where $b_{1}^{(4)}=m, b_{2}^{(4)}=b_{1}^{(4)}+\sigma, \chi_{1}^{(4)}=C$ and $\chi_{2}^{(4)}=(j+1) \chi_{1}^{(4)}$.

3.2.2. Rate-Selective RS. Dual-hop transmission incurs a pre$\log$ penalty factor of $1 / 2$. To deal with this rate loss, pure RS is considered only if it provides higher achievable rate than that of the direct $S \rightarrow D$ transmission $[10,21,35]$, that is, higher than $\log _{2}\left(1+g_{0}\right)$. Using instantaneous CSI and (32), rateselective RS chooses between direct (non-relay-assisted) and RS-assisted transmission based on the following criterion [21, equation (14)]:

$$
\mathcal{R}_{\text {sel }}=\max \left\{\frac{1}{2} \log _{2}\left(1+g_{\text {end }}\right), \log _{2}\left(1+g_{0}\right)\right\} \text {. }
$$

As shown in [21], the MGF of $g_{\text {sel }}$ can be obtained using the $\mathcal{M}_{g_{\text {end }}}(s)$ of pure RS as

$$
\mathcal{M}_{g_{\text {sel }}}(s)=\mathcal{M}_{g_{0}}(s) F_{g_{\text {end }}}(\alpha)+\mathcal{M}_{g_{\text {end }}}(s)\left[1-F_{g_{\text {end }}}(\alpha)\right] \text {, }
$$

where $\alpha=g_{0}^{2}+2 g_{0}$ is an RV with CDF given by $F_{\alpha}(x)=$ $F_{g_{0}}(\sqrt{x+1}-1)$ which can be obtained using inverse sampling [36]. By plugging (34) and (36) into (40), a closedform expression for $\mathcal{M}_{g_{\text {sel }}}(s)$ of rate-selective RS over INID Nakagami- $m$ fading channels with integer $m_{\ell}$ 's and distinct $C_{\ell}$ 's is derived as

$$
\begin{aligned}
& \mathcal{M}_{g_{\text {sel }}}(s)=\frac{C_{0}^{m_{0}}}{\left(s+C_{0}\right)^{m_{0}}}\left(\prod_{k=1}^{L} \mathcal{P}_{k}\right) \\
& \times\left\{\left\{\mathcal{Z}_{m_{0}}\left(C_{0}, \alpha\right)+\sum_{\left\{\lambda_{k}\right\}_{k=1}^{L}} \sum_{\left\{\mu_{j}\right\}_{j=1}^{k}}(-1)^{j}\left(\prod_{n=1}^{k} \frac{1-\mathcal{P}_{\lambda_{n}}}{\mathcal{P}_{\lambda_{n}}}\right)\right.\right.
\end{aligned}
$$

$$
\begin{aligned}
& \times\left\{-C_{0}^{m_{0}}\left\{\mathcal{X}_{2,1}^{(1)} \mathcal{Z}_{0}\left[\chi_{2}^{(1)}, \alpha\right]+\chi_{2}^{(1)} \mathcal{y}_{1}^{(1)}(\alpha)\right\}\right. \\
& +\sum_{\left\{v_{i}\right\}_{i=1}^{j}}\left(\prod_{s=1}^{p} \sum_{i_{\nu_{s}}=1}^{m_{\mu_{\nu_{s}}}-1} \frac{C_{\lambda_{\mu_{\nu_{s}}}^{i_{\nu_{s}}}}}{i_{\nu_{s}} !}\right) \\
& \left.\left.\times C_{0}^{m_{0}}\left(\sum_{s=1}^{p} i_{v_{s}}\right) !\left[y_{2}^{(2)}(\alpha)-\xi_{p, j} y_{2}^{(3)}(\alpha)\right]\right\}\right\} \\
& +\left\{1+\sum_{\left\{\lambda_{k}\right\}_{k=1}^{L}} \sum_{\left\{\mu_{j}\right\}_{j=1}^{k}}(-1)^{j}\left(\prod_{n=1}^{k} \frac{1-\mathcal{P}_{\lambda_{n}}}{\mathcal{P}_{\lambda_{n}}}\right)\right. \\
& \times\left\{-\left(\sum_{p=1}^{j} C_{\lambda_{\mu p}}\right)\left(s+\sum_{p=1}^{j} C_{\lambda_{\mu_{p}}}\right)^{-1}+\sum_{\left\{v_{i}\right\}_{i=1}^{j}}\left(\sum_{s=1}^{p} i_{v_{s}}\right) !\right. \\
& \left.\times\left(\prod_{s=1}^{p} \sum_{i_{v_{s}}=1}^{m_{\lambda_{\nu_{s}}}-1} \frac{C_{\lambda_{\mu_{s}}}^{i_{v_{s}}}}{i_{v_{s}} !}\right) \frac{\left[1-\xi_{p, j}\left(s+\xi_{p, j}\right)^{-1}\right]}{\left(s+\xi_{p, j}\right)^{\sum_{s=1}^{p} i_{v_{s}}}}\right\} \\
& \times\left\{1-\left(\prod_{k=1}^{L} \mathcal{P}_{k}\right)\right. \\
& \times\left\{\mathcal{Z}_{m_{0}}\left(C_{0}, \alpha\right)+\sum_{\left\{\lambda_{k}\right\}_{k=1}^{L}} \sum_{\left\{\mu_{j}\right\}_{j=1}^{k}}(-1)^{j}\left(\prod_{n=1}^{k} \frac{1-\mathcal{P}_{\lambda_{n}}}{\mathcal{P}_{\lambda_{n}}}\right)\right. \\
& \times\left\{-C_{0}^{m_{0}}\left\{\mathcal{X}_{2,1}^{(1)} \mathcal{Z}_{0}\left[\chi_{2}^{(1)}, \alpha\right]+\chi_{2}^{(1)} \mathcal{y}_{1}^{(1)}(\alpha)\right\}\right. \\
& +\sum_{\left\{\nu_{i}\right\}_{i=1}^{j}}\left(\prod_{s=1}^{p} \sum_{i_{v_{s}}=1}^{m_{\mu \nu_{s}}-1} \frac{C_{\lambda_{\mu \nu_{s}}}^{i_{\nu_{s}}}}{i_{v_{s}} !}\right) C_{0}^{m_{0}}\left(\sum_{s=1}^{p} i_{\nu_{s}}\right) ! \\
& \left.\left.\left.\left.\times\left[y_{2}^{(2)}(\alpha)-\xi_{p, j} y_{2}^{(3)}(\alpha)\right]\right\}\right\}\right\}\right\} .
\end{aligned}
$$

By plugging (35) and (38) into (40), we obtain the following closed-form expression for $\mathcal{M}_{g_{\text {sel }}}(s)$ for rate-selective RS under IID Nakagami- $m$ fading channels with integer $m$ :

$$
\begin{aligned}
& \mathcal{M}_{g_{\text {sel }}}(s)=\frac{C^{m} L \mathcal{P}^{L}}{(s+C)^{m}} \\
& \times\left\{\left\{\frac{\mathfrak{Z}_{m}(C, \alpha)}{L}+(1-\mathcal{P}) \mathfrak{Z}_{2 m}(C, \alpha)\right.\right. \\
& +\sum_{k=1}^{L-1}\left(\begin{array}{c}
L-1 \\
k
\end{array}\right) \frac{(1-\mathcal{P})^{k}}{\mathcal{P}^{k+1}}
\end{aligned}
$$




$$
\begin{aligned}
& \times\left\{\mathcal{P} \mathcal{Z}_{m}(C, \alpha)+(1-\mathcal{P}) \mathcal{Z}_{2 m}(C, \alpha)+\sum_{j=1}^{k}\left(\begin{array}{l}
k \\
j
\end{array}\right)(-1)^{j}\right. \\
& \times\left[\mathcal{P} \mathcal{Z}_{m}(C, \alpha)+\sum_{n_{1}+n_{2}+\cdots+n_{\mathrm{m}}=j}^{j}(1-\mathcal{P})\right. \\
& \left.\left.\left.\times \frac{\prod_{i=1}^{m} n_{i}^{-1} C^{2 m+\sigma}}{(m-1) ! \prod_{j=2}^{m-1}(j !)^{n_{j+1}}} y_{2}^{(4)}(\alpha)\right]\right\}\right\} \\
& +\left\{\frac{1}{L}+\frac{(1-\mathcal{P}) C^{m}}{(s+C)^{m}}+\sum_{k=1}^{L-1}\left(\begin{array}{c}
L-1 \\
k
\end{array}\right) \frac{(1-\mathcal{P})^{k}}{\mathcal{P}^{k+1}}\right. \\
& \times\left\{\mathcal{P}+\frac{(1-\mathcal{P}) C^{m}}{(s+C)^{m}}+\sum_{j=1}^{k}\left(\begin{array}{l}
k \\
j
\end{array}\right)(-1)^{j}\right. \\
& \times\left[\mathcal{P}+\sum_{n_{1}+n_{2}+\cdots+n_{m}=j}^{j} \frac{(1-\mathcal{P}) C^{m+\sigma} \prod_{i=1}^{m} n_{i}^{-1}}{\prod_{j=2}^{m-1}(j !)^{n_{j+1}}(m-1) !}\right. \\
& \left.\left.\left.\times[s+(j+1) C]^{-(m+\sigma)}\right]\right\}\right\} \\
& \times\left\{1-L \mathcal{P}^{L}\right. \\
& \times\left\{\frac{\mathfrak{Z}_{m}(C, \alpha)}{L}+(1-\mathcal{P}) \mathcal{Z}_{2 m}(C, \alpha)\right. \\
& +\sum_{k=1}^{L-1}\left(\begin{array}{c}
L-1 \\
k
\end{array}\right) \frac{(1-\mathcal{P})^{k}}{\mathcal{P}^{k+1}} \\
& \times\left\{\mathcal{P Z}_{m}(C, \alpha)+(1-\mathcal{P}) \mathcal{Z}_{2 m}(C, \alpha)+\sum_{j=1}^{k}\left(\begin{array}{l}
k \\
j
\end{array}\right)(-1)^{j}\right. \\
& \times\left[\mathcal{P} \mathcal{Z}_{m}(C, \alpha)+\sum_{n_{1}+n_{2}+\cdots+n_{m}=j}^{j}(1-\mathcal{P})\right. \\
& \left.\left.\left.\times \frac{\prod_{i=1}^{m} n_{i}^{-1} C^{2 m+\sigma} y_{2}^{(4)}(\alpha)}{(m-1) ! \prod_{j=2}^{m-1}(j !)^{n_{j+1}}}\right]\right\}\right\}\{\} \text {. }
\end{aligned}
$$

\section{Performance Analysis of ODF Relaying Schemes}

In this section, the performance of the presented ODF relaying schemes with repetitive and RS-based transmission over INID Nakagami- $m$ fading channels is analyzed. To this end, we present closed-form and analytical expressions, respectively, for the following performance metrics: (i) OP and (ii) ASEP of several modulation formats.
4.1. Repetitive Transmission. Using the closed-form expressions for $F_{\text {gend }}(x)$ and $\mathcal{M}_{\text {gend }}(s)$ presented in Section 3.1, the OP and ASEP of repetitive transmission with both MRD and $\mathrm{SD}$ are easily obtained as follows.

$O P$ : The end-to-end OP of repetitive transmission is easily obtained using $F_{g_{\text {end }}}(x)$ as

$$
P_{\text {out }}=F_{g_{\text {end }}}\left[2^{(L+1) \mathcal{R}}-1\right] .
$$

By plugging (15) and (16) into (43), closed-form expressions for the $P_{\text {out }}$ of repetitive transmission with MRD over INID Nakagami- $m$ fading channels with integer $m_{\ell}$ 's and distinct $C_{\ell}$ 's, as well as with arbitrary $m_{\ell}$ 's and $C_{\ell}=C$, for all $\ell$, are obtained [26]. Similarly, by plugging (5) into (18) and (20) into (43), we obtain a novel closed-form expression for the $P_{\text {out }}$ of repetitive transmission with SD over INID Nakagami$m$ fading channels with arbitrary values for $m_{\ell}$ 's.

ASEP: Following the MGF-based approach [34] and using the $\mathcal{M}_{g_{\text {end }}}(s)$ expressions given by (13) for repetitive transmission with MRD and (28) and (30) for repetitive transmission with SD, the ASEP of several modulation formats for the presented repetitive relaying schemes over INID Nakagami- $m$ fading channels can be easily evaluated. For example, the ASEP of noncoherent binary frequency shift keying (NBFSK) and differential binary phase shift keying (DBPSK) modulation schemes can be directly calculated from $\mathcal{M}_{g_{\text {end }}}(s)$; the average bit error rate probability (ABEP) of NBFSK is given by $\bar{P}_{b}=0.5 \mathcal{M}_{\text {gend }}(0.5)$ and of DBPSK by $\bar{P}_{b}=0.5 \mathcal{M}_{g_{\text {end }}}(1)$. For other schemes, including binary phase shift keying (BPSK), $M$-ary phase shift keying ( $M$ PSK) (It is noted that for modulation order $M>2$, gray encoding is assumed so that $\bar{P}_{s}=\bar{P}_{b} \log _{2}(M)$.), quadrature amplitude modulation ( $M$-QAM), amplitude modulation (M-AM), and differential phase shift keying ( $M$-DPSK), single integrals with finite limits and integrands composed of elementary functions (exponential and trigonometric) have to be readily evaluated via numerical integration [34]. For example, the ASEP of M-PSK is easily obtained as

$$
\bar{P}_{s}=\frac{1}{\pi} \int_{0}^{\pi-\pi / M} \mathcal{M}_{g_{\text {end }}}\left(\frac{g_{\text {PSK }}}{\sin ^{2} \varphi}\right) d \varphi,
$$

where $g_{\mathrm{PSK}}=\sin ^{2}(\pi / M)$, while for $M$-QAM, the ASEP can be evaluated as

$$
\begin{aligned}
\bar{P}_{\mathrm{s}}= & \frac{4}{\pi}\left(1-\frac{1}{\sqrt{M}}\right)\left[\int_{0}^{\pi / 2} \mathcal{M}_{g_{\text {end }}}\left(\frac{g_{\mathrm{QAM}}}{\sin ^{2} \varphi}\right) d \varphi\right. \\
& \left.-\left(1-\frac{1}{\sqrt{M}}\right) \int_{0}^{\pi / 4} \mathcal{M}_{g_{\text {end }}}\left(\frac{g_{\mathrm{QAM}}}{\sin ^{2} \varphi}\right) d \varphi\right],
\end{aligned}
$$

with $g_{\mathrm{QAM}}=3 /[2(M-1)]$.

4.2. RS-Based Transmission. The closed-form expressions for the statistics of pure and rate-selective RS presented in Section 3.2 can be used to evaluate the OP and ASEP of both RS-based schemes as follows.

$O P$ : Using the $F_{g_{\text {end }}}(x)$ expressions of (36) and (38) for INID and IID Nakagami- $m$ fading channels, respectively, 
with integer fading parameters, the end-to-end OP of pure RS is easily obtained as [21, equation (12)]

$$
P_{\text {out }}=F_{\text {gend }}\left(2^{2 \mathcal{R}}-1\right) \text {. }
$$

For rate-selective RS, a closed-form expression for $P_{\text {out }}$ over INID Nakagami- $m$ fading with arbitrary $m_{\ell}$ 's can be easily obtained by plugging (5) and (20) into [21, equation (15)] which yields

$$
\begin{aligned}
& P_{\text {out }}= \frac{\Gamma\left[m_{0}, C_{0}\left(2^{\mathcal{R}}-1\right)\right]}{\Gamma\left(m_{0}\right)} \\
& \times \prod_{k=1}^{L}\left\{\mathcal{P}_{k} u\left(2^{2 \mathcal{R}}-1\right)\right. \\
&\left.\quad+\left(1-\mathcal{P}_{k}\right) \frac{\Gamma\left[m_{k}, C_{k}\left(2^{2 \mathcal{R}}-1\right)\right]}{\Gamma\left(m_{k}\right)}\right\} .
\end{aligned}
$$

ASEP: Following the MGF-based approach and using the $\mathcal{M}_{g_{\text {end }}}(s)$ expressions for pure RS of (34) and (35) for INID and IID Nakagami- $m$ fading channels, respectively, with integer fading parameters, the ASEP of several modulation formats for pure RS can be easily calculated. Similarly, the ASEP for rate-selective RS can be easily evaluated using the $\mathcal{M}_{g_{\text {sel }}}(s)$ expressions of (41) and (42) for INID and IID Nakagami- $m$ fading channels, respectively, with integer values for the fading parameters.

\section{Numerical Results and Discussions}

The analytical expressions of the previous section have been used to evaluate the performance of ODF cooperative systems utilizing repetitive and RS-based transmission over INID Nakagami- $m$ flat fading channels. Without loss of generality, it has been assumed that $S$ transmits at a rate $\mathcal{R}=1 \mathrm{bps} / \mathrm{Hz}$ and that the fading parameters of the first and the second hop of all $L$ links are equal, that is, $m_{k}=$ $m_{L+k}$ for all $k$, as well as $\bar{\gamma}_{k}=\bar{\gamma}_{L+k}$ for all $k$. Moreover, for the performance evaluation results, we have considered the exponentially power decaying profile $\bar{\gamma}_{k}=\bar{\gamma}_{0} \exp (-\ell \delta)$ with $\delta$ being the power decaying factor. Clearly, when ID fading is considered, $\delta=0$ and $\bar{\gamma}_{k}=\bar{\gamma}_{0}=\bar{\gamma}$ for all $k$. In all figures that follow, analytical results for the $P_{\text {out }}$ and $\bar{P}_{\mathrm{b}}$ match perfectly with the results obtained by means of Monte Carlo simulations, validating our analysis.

Figure 2 plots the $P_{\text {out }}$ of the considered relaying schemes with both repetitive and RS-based transmission as a function of $L$ for average transmit SNR $\bar{\gamma}_{0}=5 \mathrm{~dB}$ over IID Nakagami$m$ fading channels with different values of $m$. As clearly shown, $P_{\text {out }}$ improves with increasing $L$ for both RS-based transmission schemes whereas it degrades severely for both repetitive ones. In particular, utilizing repetitive transmission for $\bar{\gamma}_{0}=5 \mathrm{~dB}$ is unavailing for $L \geq 3$ irrespective of the fading conditions. As for RS-based transmission, the gains from RS diminish as $m$ increases; the smaller the value of $m$, the greater the gain from relaying on $P_{\text {out }}$. Furthermore, pure RS is inefficient when $L$ is small, and rate-selective RS results in significant gains on $P_{\text {out }}$ over the pure one as $m$ decreases.

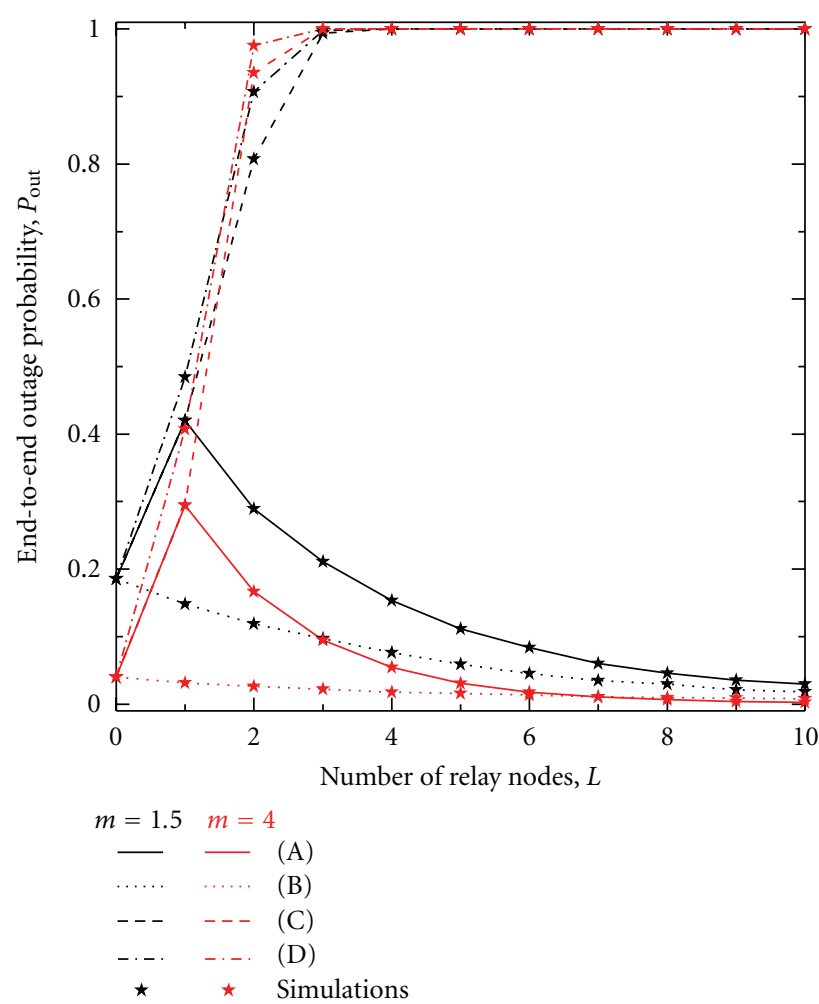

Figure 2: End-to-end OP, $P_{\text {out }}$, versus the number of relay nodes, $L$, for average transmit SNR $\bar{\gamma}_{0}=5 \mathrm{~dB}$ over IID Nakagami$m$ fading channels with different values of $m$ : (A) pure RS, (B) rate-selective RS, (C) repetitive transmission with MRD, and (D) repetitive transmission with $\mathrm{SD}$.

The impact of increasing $L$ on the $P_{\text {out }}$ performance of all four relaying schemes is also demonstrated in Figure 3 for different values of $\bar{\gamma}_{0}$ and IID Rayleigh fading conditions. As shown from this figure and Figure 2 for both repetitive and RS-based transmission, $P_{\text {out }}$ degrades with decreasing $\bar{\gamma}_{0}$ and/or $m$. For example, although for $\bar{\gamma}_{0}=5 \mathrm{~dB}$ repetitive transmission does not benefit from relaying for $L \geq 3$, this happens for $\bar{\gamma}_{0}=0 \mathrm{~dB}$ when $L \geq 2$. Moreover, for $\bar{\gamma}_{0}=0 \mathrm{~dB}$ the improvement on the $P_{\text {out }}$ of pure RS with increasing $L$ is very small, while $P_{\text {out }}$ performance of rate-selective RS does not vary with $L$. It turns out that the smaller the $\bar{\gamma}_{0}$, the smaller the gains from relaying as fading conditions become more severe and transmit SNR reduces.

In Figure 4 , the decoding probability $1-\mathcal{P}_{k}$ of $(8)$ is plotted versus $L$ for both repetitive and RS-based transmission over IID Nakagami- $m$ fading conditions for the sourceto-relay channels with different values of $m$ and $\bar{\gamma}_{L+k}=\bar{\gamma}$ for all $k, L$. As expected, decreasing $m$ and/or $\bar{\gamma}$ reduces the decoding probability. Moreover, this probability is severely degraded with increasing $L$ for repetitive transmission, whereas it remains unchanged with increasing $L$ for the RSbased one. The $P_{\text {out }}$ performance of pure and rate-selective RS is depicted in Figure 5 versus $\bar{\gamma}_{0}$ for $L=2$ relays over INID Nakagami- $m$ fading channels with different values of $m_{0}, m_{1}$, and $m_{2}$. As expected, for both RS-based schemes, $P_{\text {out }}$ improves with increasing $\bar{\gamma}_{0}$ and/or any of the fading 


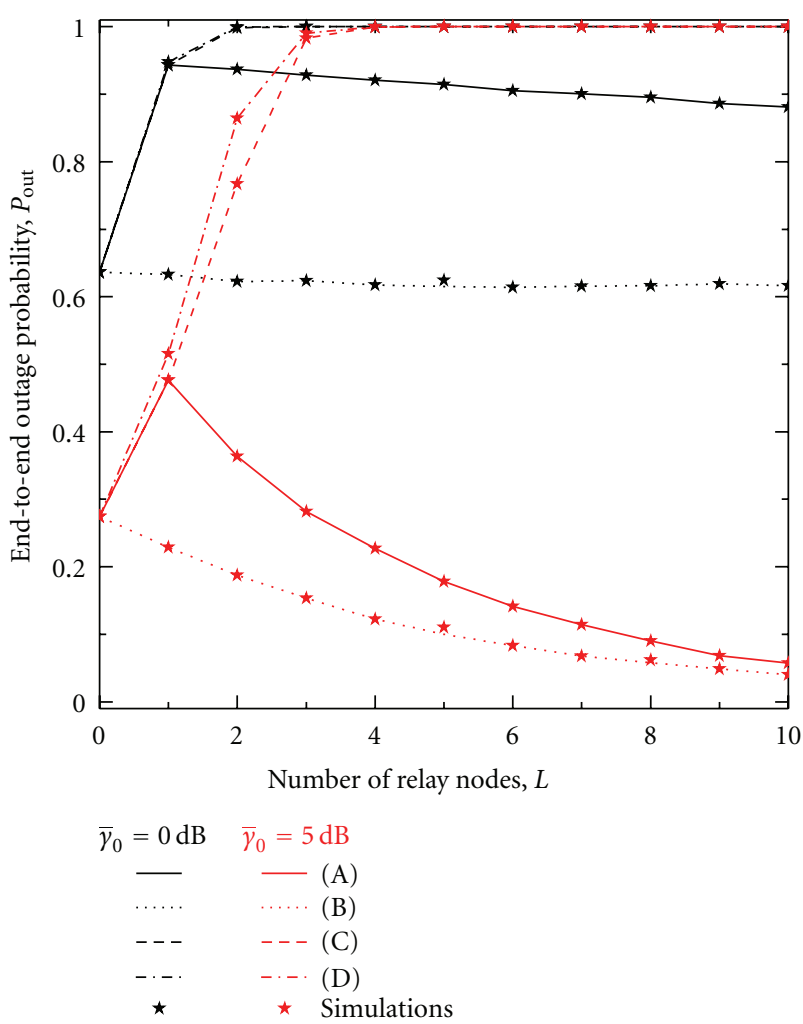

FIgUre 3: End-to-end OP, $P_{\text {out }}$, versus the number of relay nodes, $L$, for different average transmit SNRs over IID Rayleigh fading channels: (A) pure RS, (B) rate-selective RS, (C) repetitive transmission with MRD and (D) repetitive transmission with SD.

parameters. More importantly, it is shown that, as the fading conditions of the relay-to-destination channels become more favorable than those of the direct source to destination channel, RS-based transmission improves $P_{\text {out }}$. On the contrary, whenever the fading conditions of the relay-todestination channels are similar to the direct one, non-relayassisted transmission results in lower $P_{\text {out }}$ than RS-based one.

In Figure 6 , the $\bar{P}_{b}$ performance of square 4 -QAM is plotted as a function of $L$ over IID Nakagami- $m$ fading channels with different values of $m$. The average SNR per bit is assumed to be $\bar{\gamma}_{b}=5 \mathrm{~dB}$ for all second hop channels and $\bar{\gamma}_{0}=0 \mathrm{~dB}$. It is clearly shown that the $\bar{P}_{b}$ of RSbased transmission improves with increasing $L$ and/or $m$, whereas for both repetitive schemes, although $\bar{P}_{b}$ improves with increasing $m$, it does not benefit from increasing $L$. Interestingly, for RS-based transmission, as $L$ and/or $m$ increase, the $\bar{P}_{b}$ improvement of pure compared with rateselective RS increases; as shown by (39), rate-selective RS might choose the direct transmission even in cases where the received SNR from the best relay is larger than that from the source node. Assuming IID Rayleigh fading conditions, Figure 7 plots $\bar{P}_{b}$ for DBPSK versus $L$ for all considered relaying schemes and for different values of $\bar{\gamma}_{0}$. As shown in this figure and Figure 6, for both repetitive and RS-based transmission $\bar{P}_{b}$ improves with increasing $\bar{\gamma}_{0}$ and/or $m$. It was shown in Figure 4 that more favorable fading conditions

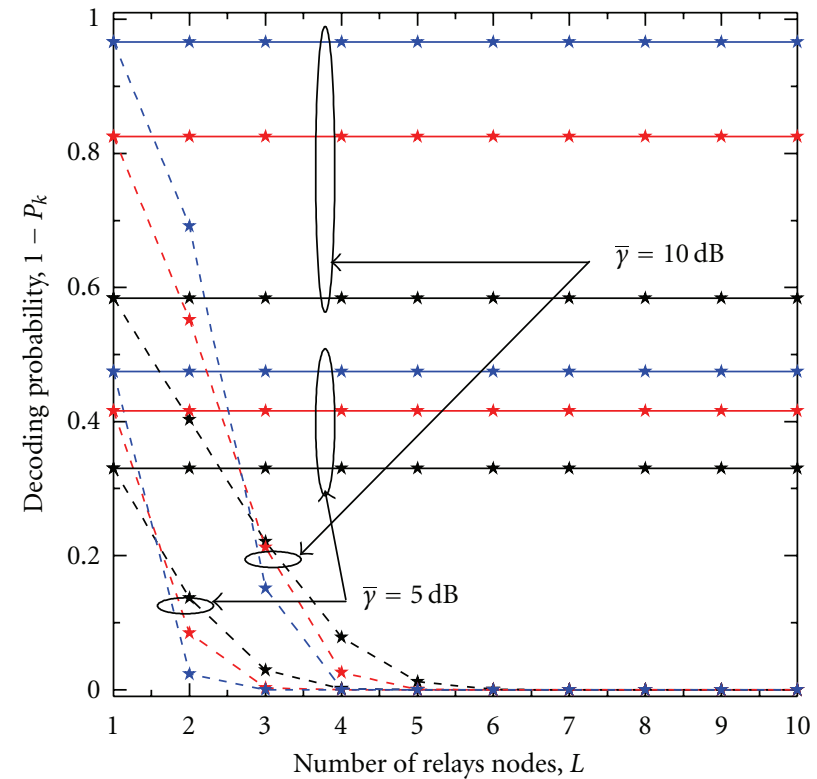

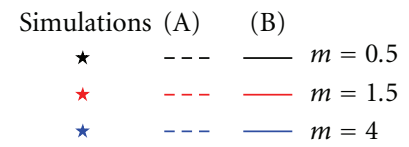

FIGURE 4: Decoding probability, $1-\mathcal{P}_{k}$, versus the number of relay nodes, $L$, over IID Nakagami- $m$ fading channels with different values of $m$ and $\bar{\gamma}_{L+k}=\bar{\gamma}$ for all $k, L$ : (A) repetitive and (B) RS-based transmission.

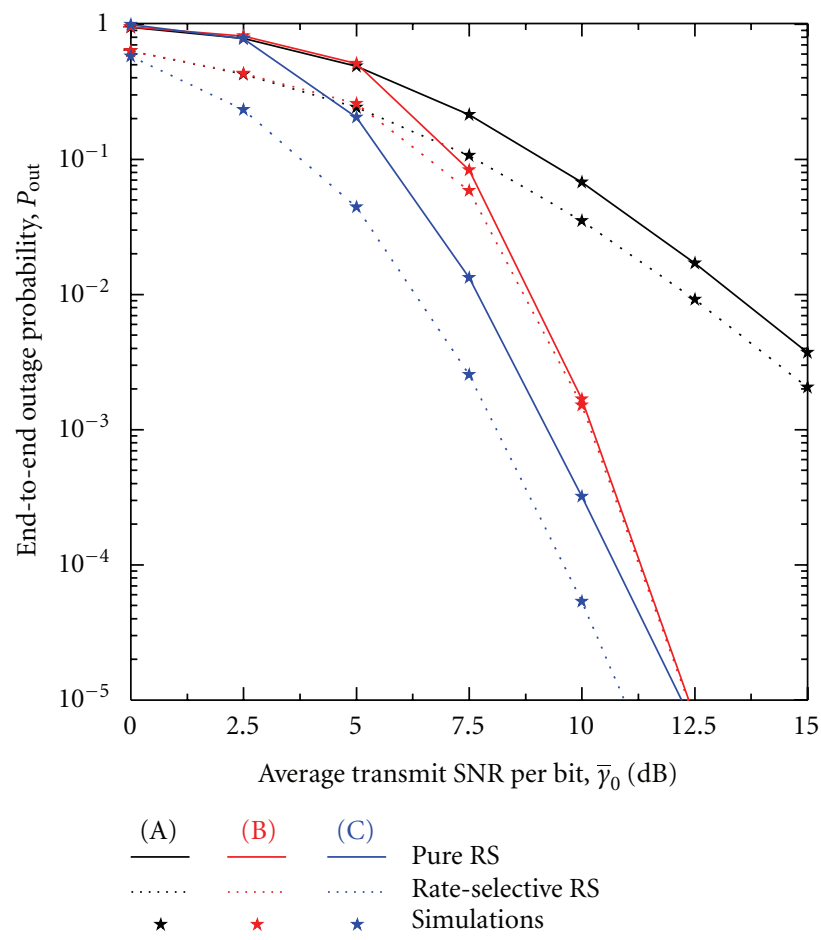

FIGURE 5: End-to-end OP, $P_{\text {out }}$, of RS versus the average transmit SNR per bit, $\bar{\gamma}_{0}$, for $L=2$ relay nodes over INID Nakagami- $m$ fading channels: (A) $m_{0}=m_{1}=m_{2}=1$ and $\delta=0.3$, (B) $m_{0}=1$, $m_{1}=m_{2}=6$, and $\delta=0.3$, and (C) $m_{0}=m_{1}=m_{2}=3$ and $\delta=0$. 


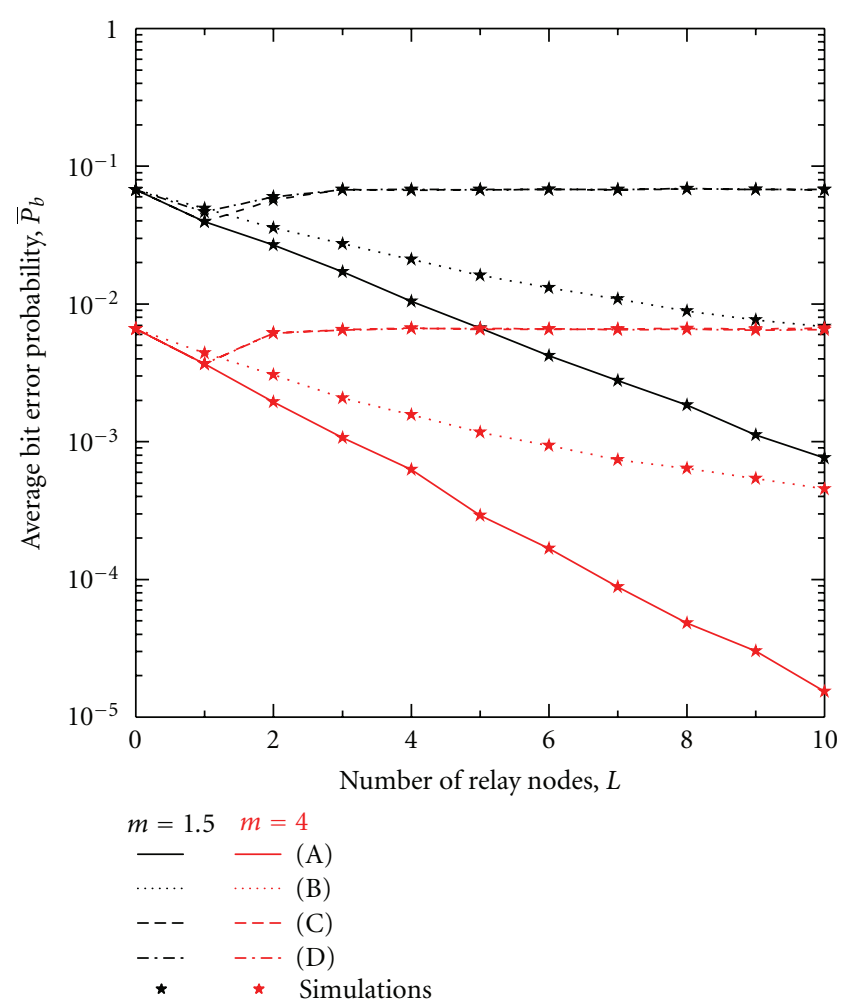

Figure 6: ABEP, $\bar{P}_{\mathrm{b}}$, of square 4-QAM versus the number of relay nodes, $L$, for average transmit SNR $\bar{\gamma}_{\mathrm{b}}=5 \mathrm{~dB}$ and $\bar{\gamma}_{0}=0 \mathrm{~dB}$ over IID Nakagami- $m$ fading channels with different values of $m$ : (A) pure RS, (B) rate-selective RS, (C) repetitive transmission with $\mathrm{MRD}$, and (D) repetitive transmission with SD.

and larger $\bar{\gamma}_{0}$ increase the decoding probability; thus, both repetitive transmission schemes become unavailing for larger $L$, whereas the gains from RS increase. The $\bar{P}_{b}$ performance of pure and rate-selective RS is depicted in Figure 8 versus $\bar{\gamma}_{b}$ for DBPSK over INID Nakagami- $m$ fading channels with different values for the fading parameters with $L=1$, 2 , and 3 relay nodes $\left(\bar{\gamma}_{0}=0 \mathrm{~dB}\right)$. Clearly, for both RSbased transmission schemes, $\bar{P}_{b}$ improves with increasing $\bar{\gamma}_{b}$ and/or $m$ and/or $L$. It is shown that as $\bar{\gamma}_{b}$ increases and as the fading conditions of the relay-to-destination channels become more favorable than those of the direct source to destination channel, RS-based transmission results in larger $\bar{P}_{b}$ improvement. More importantly, even for strong sourceto-destination channels, as $\bar{\gamma}_{b}$ increases, the improvement on $\bar{P}_{b}$ with RS-based transmission becomes larger than that of the non-relay-assisted one.

\section{Conclusion}

Cooperative diversity is a very promising avenue for future wireless communications. However, it is necessary to investigate how relays should be utilized in order to achieve certain objectives. In this paper, we presented a general analytical framework for modeling and evaluating the performance of four DF relaying schemes under INID Nakagami- $m$ fading

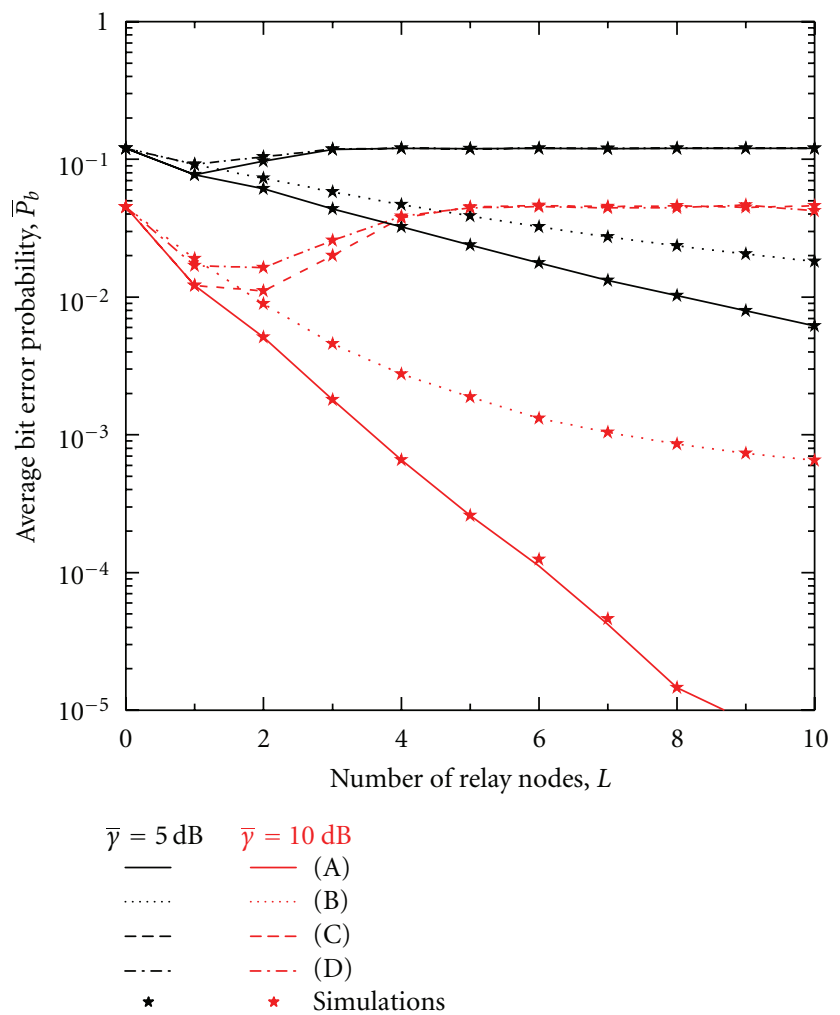

Figure 7: ABEP, $\bar{P}_{\mathrm{b}}$, of DBPSK versus the number of relay nodes, $L$, for different average transmit SNRs per bit and $\bar{\gamma}_{0}=0 \mathrm{~dB}$, over IID Rayleigh fading channels: (A) pure RS, (B) rate-selective RS, (C) repetitive transmission with $\mathrm{MRD}$, and (D) repetitive transmission with SD.

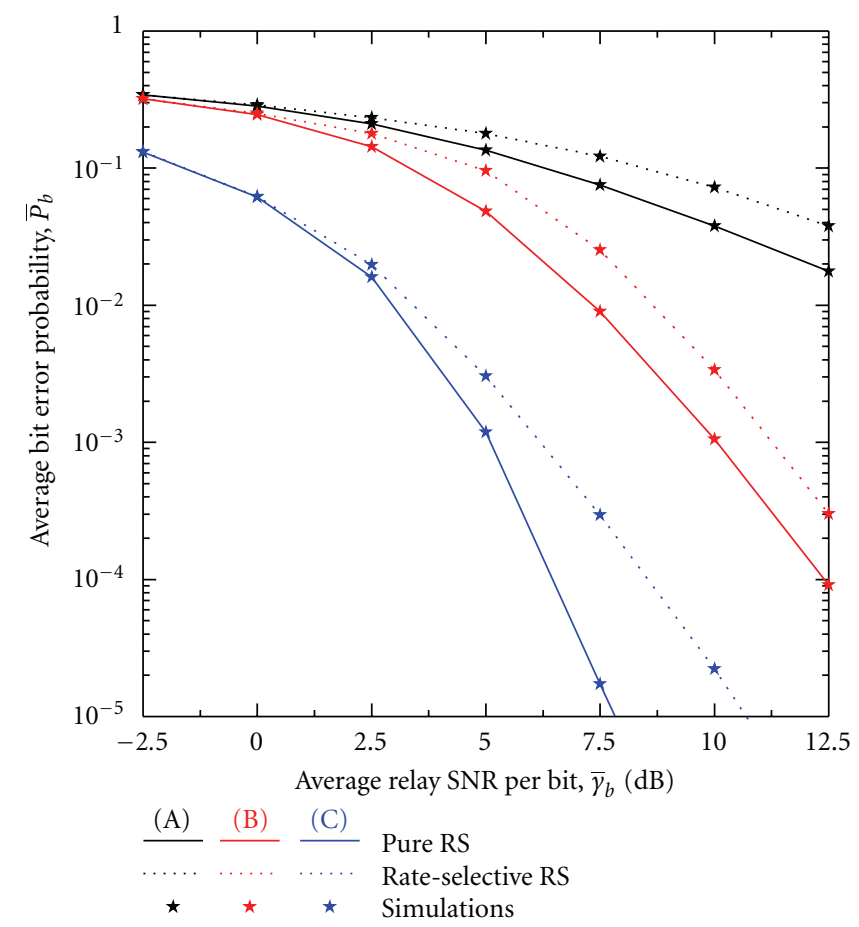

FIGURE 8: ABEP, $\bar{P}_{b}$, of DBPSK for RS versus the average relay SNR per bit, $\bar{\gamma}_{b}$, and for $\bar{\gamma}_{0}=0 \mathrm{~dB}$ over INID Nakagami- $m$ fading channels: (A) $L=1, m_{0}=0.5, m_{1}=1$, and $\delta=0.1$, (B) $L=2, m_{0}=1$, $m_{1}=m_{2}=2$, and $\delta=0$, and (C) $L=3, m_{\ell}=3$ for $\ell=0,1,2$, and 3 and $\delta=0$. 
channels. More specifically, we obtained closed-form expressions for the OP and the ASEP performance of repetitive transmission when SD is employed at the destination node and presented a comparative performance study in terms of OP and ASEP of all considered DF schemes. We concluded that RS performs better than repetitive transmission when CSI for RS is perfect. Furthermore, it was shown that relaying should be switched off when the source-to-destination direct link is sufficiently strong and the aim is minimizing OP. However, in terms of ASEP, relaying was shown always to be beneficial.

\section{References}

[1] G. J. Foschini and M. J. Gans, "On limits of wireless communications in a fading environment when using multiple antennas," Wireless Personal Communications, vol. 6, no. 3, pp. 311-335, 1998.

[2] E. Biglieri, R. Calderbank, A. Constantinides, A. Goldsmith, A. Paulraj, and H. V. Poor, MIMO Wireless Communications, Cambridge University Press, Cambridge, UK, 1st edition, 2007.

[3] G. C. Alexandropoulos, Performance study of space diversity receivers over correlated fading channels, Ph.D. dissertation, University of Patras, Rio-Patras, Greece, 2010.

[4] J. N. Laneman and G. W. Wornell, "Distributed space-timecoded protocols for exploiting cooperative diversity in wireless networks," IEEE Transactions on Information Theory, vol. 49, no. 10 , pp. 2415-2425, 2003.

[5] A. Sendonaris, E. Erkip, and B. Aazhang, "User coorperation diversity-part I: system description," IEEE Transactions on Communications, vol. 51, no. 11, pp. 1927-1938, 2003.

[6] A. Sendonaris, E. Erkip, and B. Aazhang, "User coorperation diversity-part II: implementation aspects and performance analysis," IEEE Transactions on Communications, vol. 51, no. 11, pp. 1939-1948, 2003.

[7] A. Papadogiannis, Systems and techniques for multicell-MIMO and cooperative relaying in wireless networks, Ph.D. dissertation, Telecom ParisTech, Paris, France, 2009.

[8] A. Papadogiannis, G. C. Alexandropoulos, A. G. Burr, and D. Grace, "Bringing mobile relays for wireless access networks into practice-Learning when to relay," IET Communications, to appear.

[9] A. Bletsas, A. Khisti, D. P. Reed, and A. Lippman, "A simple cooperative diversity method based on network path selection," IEEE Journal on Selected Areas in Communications, vol. 24, no. 8, pp. 659-672, 2006.

[10] A. Papadogiannis, E. Hardouin, A. Saadani, D. Gesbert, and P. Layec, "A novel framework for the utilization of dynamic relays in cellular networks," in Proceedings of the IEEE ASIMOLAR, pp. 975-979, Pacific Grove, Calif, USA, 2008.

[11] A. Papadogiannis, A. Saadani, and E. Hardouin, "Exploiting dynamic relays with limited overhead in cellular systems," in Proceedings of the IEEE Globecom Workshops, (Gc Workshops '09), Hawaii, USA, 2009.

[12] M. O. Hasna and M. S. Alouini, "End-to-end performance of transmission systems with relays over Rayleigh-fading channels," IEEE Transactions on Wireless Communications, vol. 2, no. 6, pp. 1126-1131, 2003.

[13] A. Stefanov and E. Erkip, "Cooperative space-time coding for wireless networks," IEEE Transactions on Communications, vol. 53, no. 11, pp. 1804-1809, 2005.
[14] M. Elfituri, W. Hamouda, and A. Ghrayeb, "Outage probability analysis of distributed coded cooperation for relay channels," in Proceedings of the IEEE International Symposium on Personal, Indoor and Mobile Radio Communications, (PIMRC '07), Athens, Greece, 2007.

[15] A. Nosratinia and T. E. Hunter, "Grouping and partner selection in cooperative wireless networks," IEEE Journal on Selected Areas in Communications, vol. 25, no. 2, pp. 369-378, 2007.

[16] M. Elfituri, W. Hamouda, and A. Ghrayeb, "Performance analysis of a new transmission scheme for multi-relay channels," in Proceedings of the IEEE Workshop on Signal Processing Systems Design and Implementation, (SIPS '06), pp. 34-38, 2006.

[17] M. Elfituri, W. Hamouda, and A. Ghrayeb, "Distributed coded cooperation for relay channels operating in the decodeand-forward mode," in Proceedings of the IEEE International Conference on Communications, (ICC '08), pp. 4586-4590, Beijing, China, 2008.

[18] M. Elfituri, A. Ghrayeb, and W. Hamouda, "Antenna/relay selection for coded wireless cooperative networks," in Proceedings of the IEEE International Conference on Communications, (ICC '08), pp. 840-844, Beijing, China, 2008.

[19] S. I. Hussain, M. O. Hasna, and M. S. Alouini, "Performance analysis of best relay selection scheme for fixed gain cooperative networks in non-identical Nakagami-m channels," in Proceedings of the 7th International Symposium on Wireless Communication Systems, (ISWCS'10), pp. 255-259, York, UK, 2010.

[20] K. Yan, J. Jiang, Y. G. Wang, and H. T. Liu, "Outage probability of selection cooperation with MRC in Nakagami- $m$ fading channels," IEEE Signal Processing Letters, vol. 16, no. 12, pp. 1031-1034, 2009.

[21] G. C. Alexandropoulos, A. Papadogiannis, and K. Berberidis, "Performance analysis of cooperative networks with relay selection over Nakagami- $m$ fading channels," IEEE Signal Processing Letters, vol. 17, no. 5, pp. 441-444, 2010.

[22] N. C. Beaulieu and J. Hu, "A closed-form expression for the outage probability of decode-and-forward relaying in dissimilar Rayleigh fading channels," IEEE Communications Letters, vol. 10, no. 12, pp. 813-815, 2006.

[23] J. Hu and N. C. Beaulieu, "Performance analysis of decodeand-forward relaying with selection combining," IEEE Communications Letters, vol. 11, no. 6, pp. 489-491, 2007.

[24] E. Beres and R. Adve, "Selection cooperation in multisource cooperative networks," IEEE Transactions on Wireless Communications, vol. 7, no. 1, pp. 118-127, 2008.

[25] F. Xu, F. C. M. Lau, Q. F. Zhou, and D.-W. Yue, "Outage performance of cooperative communication systems using opportunistic relaying and selection combining receiver," IEEE Signal Processing Letters, vol. 16, no. 4, pp. 237-240, 2009.

[26] C. K. Datsikas, N. C. Sagias, F. I. Lazarakis, and G. S. Tombras, "Outage analysis of decode-and-forward relaying over Nakagami-m fading channels," IEEE Signal Processing Letters, vol. 15, no. 1, pp. 41-44, 2008.

[27] A. Papadogiannis and G. C. Alexandropoulos, "System level performance evaluation of dynamic relays in cellular networks over Nakagami- $m$ fading channels," in Proceedings of the IEEE International Symposium on Personal, Indoor and Mobile Radio Communications, (PIMRC '09), Tokyo, Japan, 2009.

[28] G. C. Alexandropoulos, A. Papadogiannis, and K. Berberidis, "Relay selection vs. repetitive transmission cooperation: analysis under Nakagami-m fading," in Proceedings of the IEEE International Symposium on Personal, Indoor and Mobile Radio 
Communications, (PIMRC'10), pp. 140-144, Istanbul, Turkey, 2010.

[29] T. Q. Duong, G. C. Alexandropoulos, H.-J. Zepernick, and T. A. Tsiftsis, "Orthogonal space-time block codes with CSIassisted amplify-and forward relaying in correlated Nakagami$m$ fading channels," IEEE Transactions on Vehicular Technology, vol. 60, no. 3, pp. 882-889, 2011.

[30] T. Q. Duong, G. C. Alexandropoulos, T. A. Tsiftsis, and H.-J. Zepernick, "Outage probability of MIMO AF relay networks over Nakagami- $m$ fading channels," Electronics Letters, vol. 46, no. 17, pp. 1229-1231, 2011.

[31] P. C. Sofotasios, G. C. Alexandropoulos, A. Papadogiannis, Q. T. Duong, and S. Freear, "Error probability of DF relay selection: Sensitivity of the Nakagami fading parameter," submitted to Electronics Letters.

[32] I. S. Gradshteyn and I. M. Ryzhik, Table of Integrals, Series, and Products, Academic Press, New York, NY, USA, 6th edition, 2000.

[33] M. Nakagami, "The $m$-distribution-a general formula of intensity distribution of rapid fading," in Statistical Methods in Radio Wave Propagation, W. G. Hoffman, Ed., pp. 3-36, Permagon Press, Oxford, UK, 1960.

[34] M. K. Simon and M.-S. Alouini, Digital Communication over Fading Channels, John Wiley \& Sons, New York, NY, USA, 2nd edition, 2005.

[35] K. Woradit, T. Q. S. Quek, W. Suwansantisuk, H. Wymeersch, L. Wuttisittikulkij, and M. Z. Win, "Outage behavior of cooperative diversity with relay selection," in Proceedings of the IEEE Global Telecommunications Conference (GLOBECOM '08), pp. 966-970, New Orleans, LA, USA, 2008.

[36] W. G. Cochran, Sampling Techniques, John Wiley \& Sons, New York, NY, USA, 3rd edition, 1977. 

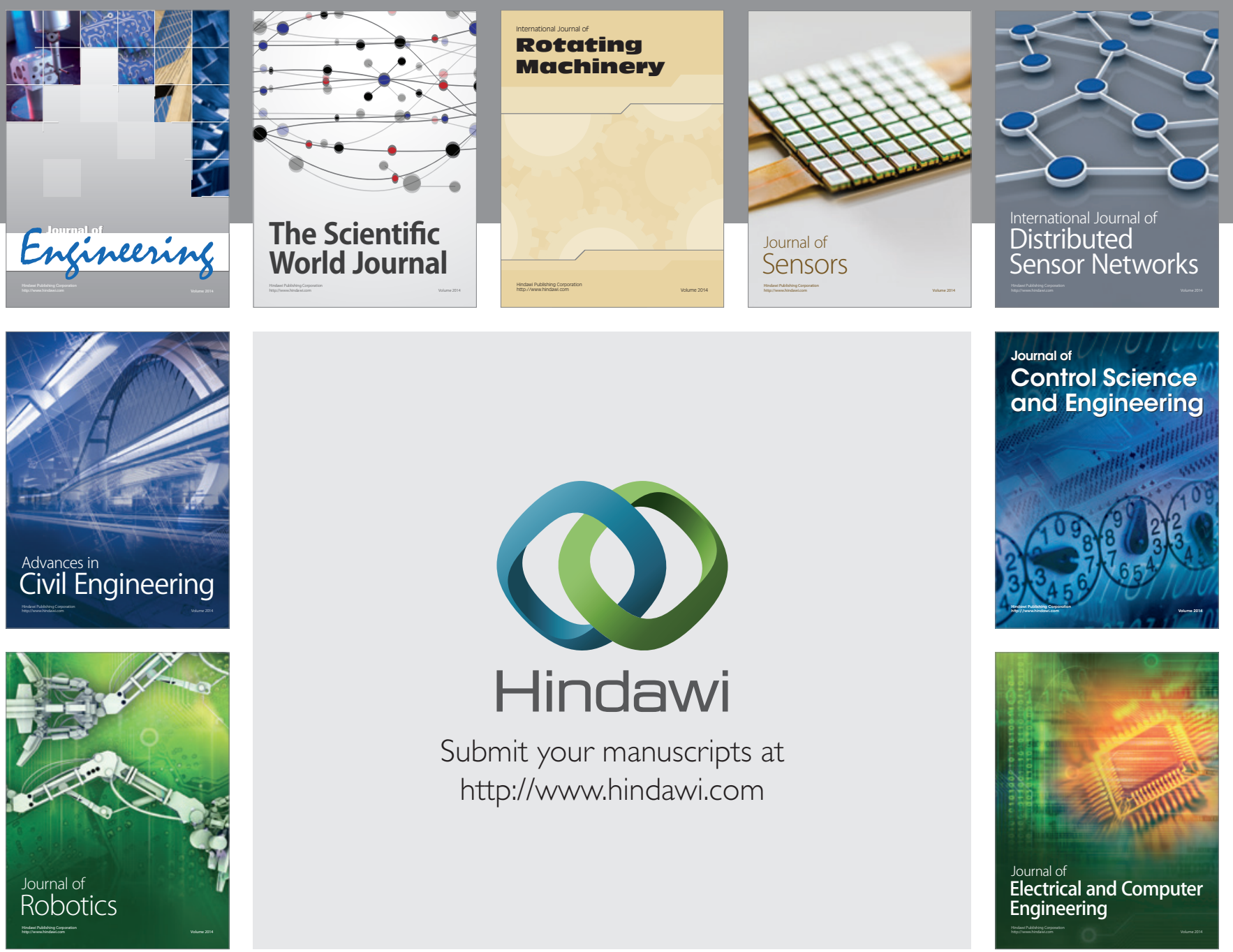

Submit your manuscripts at

http://www.hindawi.com
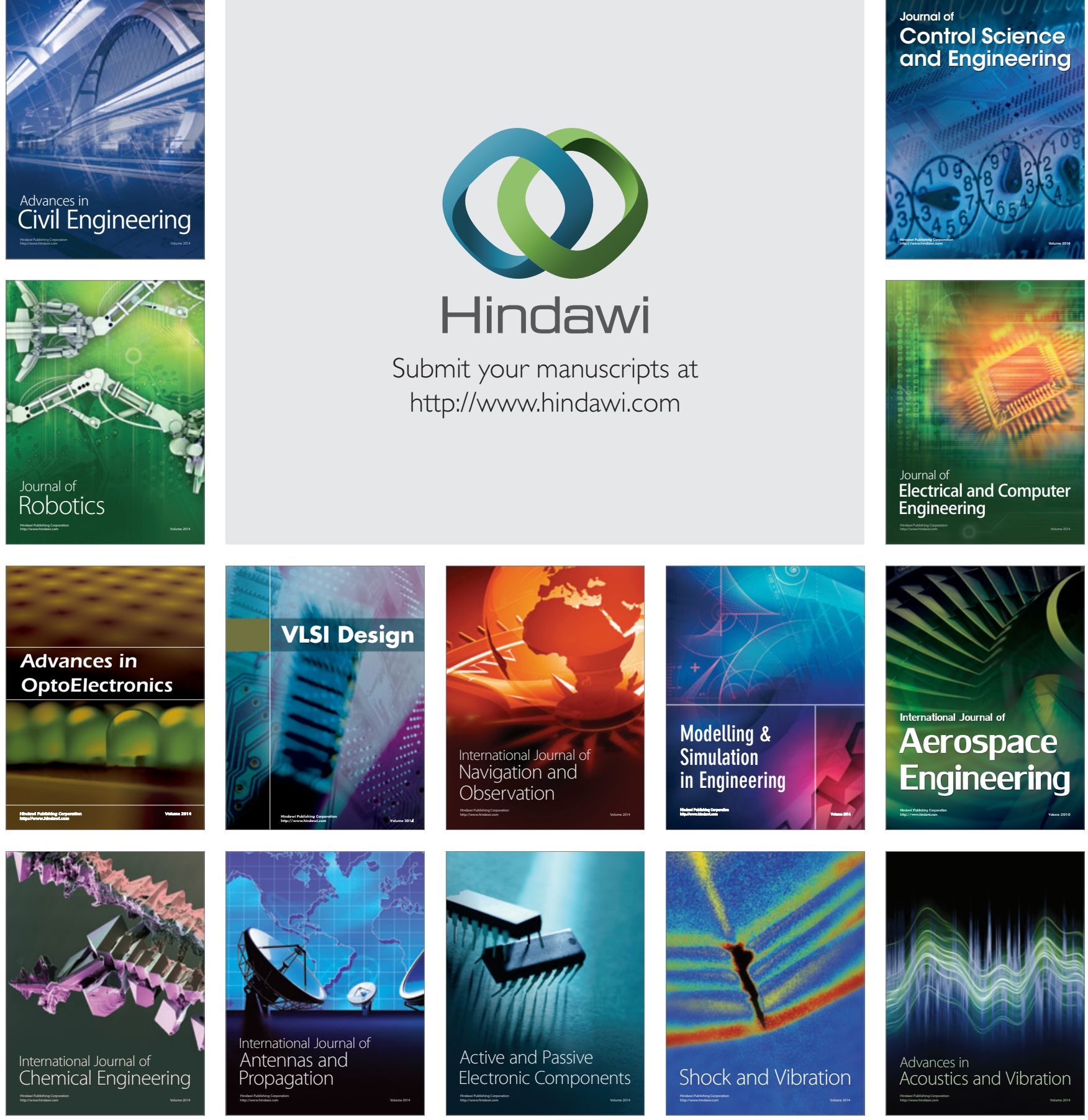